\title{
Global Dynamics of an SVEIR Model with Age-Dependent Vaccination, Infection, and Latency
}

\author{
Rodrigue Yves M'pika Massoukou ${ }^{\mathbb{D}},{ }^{1}$ Suares Clovis Oukouomi Noutchie $\mathbb{D}^{1}{ }^{1}$ \\ and Richard Guiem ${ }^{1,2}$ \\ ${ }^{1}$ School of Mathematical and Statistical Sciences, North-West University, Mafikeng campus, Private Bag X2046, \\ Mmabatho 2735, South Africa \\ ${ }^{2}$ National Advanced School of Engineering of Maroua, University of Maroua, P.O. Box 46, Maroua, Cameroon
}

Correspondence should be addressed to Rodrigue Yves M'pika Massoukou; rodrigue@aims.ac.za

Received 9 April 2018; Revised 26 June 2018; Accepted 18 July 2018; Published 14 August 2018

Academic Editor: Jaume Giné

Copyright (C) 2018 Rodrigue Yves M'pika Massoukou et al. This is an open access article distributed under the Creative Commons Attribution License, which permits unrestricted use, distribution, and reproduction in any medium, provided the original work is properly cited.

\begin{abstract}
Vaccine-induced protection is substantial to control, prevent, and reduce the spread of infectious diseases and to get rid of infectious diseases. In this paper, we propose an SVEIR epidemic model with age-dependent vaccination, latency, and infection. The model also considers that the waning vaccine-induced immunity depends on vaccination age and the vaccinated individuals fall back to the susceptible class after losing immunity. The model is a coupled system of (hyperbolic) partial differential equations with ordinary differential equations. The global dynamics of the model is established through construction of appropriate Lyapunov functionals and application of Lasalle's invariance principle. As a result, the global stability of the infection-free equilibrium and endemic equilibrium is obtained and is fully determined by the basic reproduction number $\mathfrak{R}_{0}$.
\end{abstract}

\section{Introduction}

Protection induced by vaccines plays a significant role in preventing and reducing the transmission of infectious diseases. One of the greatest successful events of vaccination is illustrated through the eradication of small-pox. It is reported in [1] that the case of small-pox was last recorded in 1977. Immunity conveyed by vaccination depends on different vaccines and vaccination policies-lifelong immunity occurs for certain vaccines while immunization period is induced by some vaccines. However, waning vaccineinduced immunity takes place (naturally) after immunization process. It is reported in [2] that a significant decay in the proportion of chicken pox took place in US in 1995 after conducting a universal vaccination campaign. But, surprisingly new cases of chicken pox appeared mainly in highly vaccinated school communities in US. Some studies were conducted and revealed waning vaccine-induced immunity in children under protection induced by vaccine.
Moreover, this was also investigated in [3-5] and it was proved that such waning of immunity is attached to the time since vaccination and the age at vaccination. In this regard, it was published in $[6,7]$ that the time of vaccineinduced immunity depends on individuals features and vaccine age.

From the abovementioned statements and citations, we see that it is necessary to associate waning vaccine-induced immunity with vaccination in infectious disease models and it is interesting to investigate the impact of waning vaccineinduced immunity on the dynamics of infectious diseases. Many mathematical models on vaccination have been already investigated and, to cite a few of them, see $[2,8-16]$. Some of the above-cited model considered either age-dependent vaccination, while some did not.

Despite vaccination age-structure being the main and appropriate feature required in the dynamics of infectious diseases with waning induced-vaccine immunity, most of epidemiological models with vaccination including waning 
induced-vaccine immunity were studied after assuming a constant rate of immunity loss (to name a few, see $[9,10$, 15]). Age-dependent vaccination was considered in some epidemiological models studied recently in $[1,2,17-20]$. However, some of these works considered either waning vaccine-induced immunity or not, either vaccine-agedependent waning vaccine-induced immunity or not, either age-dependent latency or not, either age-dependent relapse or not, and either age-dependent infection or not. In [17] an SVIR epidemic model with continuous age-dependent vaccination was formulated to establish the global stability of equilibria. In [18] an SVIJS epidemic model with agedependent vaccination was considered to study the asymptotical behavior of the equilibria, after assuming that agedependent vaccine-induced immunity decays with time after vaccination. In [19] an SVIS epidemic model with agedependent vaccination and vaccine-age-dependent waning vaccine-induced immunity and treatment was formulated to investigate backward bifurcations. In [2] an SVIR epidemic model with vaccination age was considered to establish global stability of equilibria, after assuming that vaccine-induced immunity decays with time after vaccination. In [20] an SVEIR epidemic model with age-dependence vaccination, latency, and relapse was formulated to establish the global stability of the equilibria. In [1] a multigroup SVEIR epidemic model with latent class and vaccination age was formulated to study global stability of equilibria, after assuming that vaccine-induced immunity decays with time after vaccination and likewise in [17-19].

Recently, in [14] an SVEIR epidemic model with continuous age-structure in the latent and infectious classes and without continuous age-structure in the vaccinated class was formulated to prove the global stability of equilibria, while in [20] an SVEIR epidemic model with continuous age-structure in the latent, infectious, and recovered classes and with vaccine-age-dependent waning vaccine-induced immunity was formulated. Moreover, in [14] the latency and infection ages are denoted by the same variable $a$. Similarly, in [20] the latency, relapse, and vaccination ages are denoted by the same variable $a$. In spite of this, to the best of our knowledge, the global dynamics of an SVEIR epidemic model with continuous age-structure in latency, infection, vaccination, and vaccine-age-dependence waning vaccineinduced immunity has not yet been neither considered nor investigated using the approach of Lyapunov functionals. The aim of this work is to fill this gap by investigating the global dynamics of an SVEIR epidemic model as above defined. Motivated by $[14,20]$, we propose a new SVEIR epidemic model originated from an existing SVEIR formulated in [1], by considering continuous age-structure in latency and infection in addition to age-dependence vaccination and vaccine-age-dependence waning vaccine-induced immunity (which the authors took into account in [1]). However, the latency, infection, and vaccination ages are all denoted by $a$, as in $[14,20]$. Moreover, in this paper, we also consider a more significant incidence rate (taking into account transmission by both age-mates infective individuals and infective individuals of any age) of the form

$$
\begin{aligned}
& S(t) \int_{0}^{\infty}\left(K_{0}(a) i(a, t)\right. \\
& \left.\quad+\int_{0}^{\infty} K\left(a, a^{\prime}\right) i\left(a^{\prime}, t\right) d a^{\prime}\right) d a,
\end{aligned}
$$

where $K_{0}(a)$ and $K\left(a, a^{\prime}\right)$ are defined below, instead of the classical incidence rate of the form

$$
S(t) \int_{0}^{\infty} \beta(a) i(a, t) d a,
$$

where $\beta(a)$ denotes the coefficient of disease transmission from infective individual, with infection age $a$, to susceptible individual. The latter is considered in the references therein where continuous age-structure in infection is taken into account.

The model splits the total population into five epidemiological compartments, namely, the susceptible compartment, the vaccinated compartment, the latent compartment, infected compartment, and the removed compartment. Let $S(t)$ and $R(t)$ be the number of individuals in the susceptible and removed compartments at time $t$, respectively. Let $v(a, t)$, $e(a, t)$, and $i(a, t)$ be the density of vaccinated, (latently) infected, and (actively) infected individuals with vaccination, latency, and infection age $a$ at time $t$, respectively. It follows that $V(t), E(t)$, and $I(t)$ defined by

$$
\begin{gathered}
V(t)=\int_{0}^{\infty} v(a, t) d a, \\
E(t)=\int_{0}^{\infty} e(a, t) d a, \\
I(t)=\int_{0}^{\infty} i(a, t) d a,
\end{gathered}
$$

are the number of individuals in the vaccinated, latent, and infected compartments, respectively.

The model to be investigated consists of a hybrid system of nonlinear coupled ordinary differential equations and partial differential equations of the form

$$
\begin{aligned}
& \frac{\mathrm{d}}{\mathrm{d} t} S(t)=\Lambda-\left(\nu+\mu^{0}\right) S(t)+\int_{0}^{\infty} \alpha(a) v(a, t) d a \\
& -S(t) \int_{0}^{\infty}\left(K_{0}(a) i(a, t)\right. \\
& \left.\quad+\int_{0}^{\infty} K\left(a, a^{\prime}\right) i\left(a^{\prime}, t\right) d a^{\prime}\right) d a \\
& \frac{\partial}{\partial t} v(a, t)=-\frac{\partial}{\partial a} v(a, t)-\eta(a) v(a, t) \\
& \frac{\partial}{\partial t} e(a, t)=-\frac{\partial}{\partial a} e(a, t)-\varrho(a) e(a, t) \\
& \frac{\partial}{\partial t} i(a, t)=-\frac{\partial}{\partial a} i(a, t)-\sigma(a) i(a, t) \\
& \frac{\mathrm{d}}{\mathrm{d} t} R(t)=\int_{0}^{\infty} \gamma(a) i(a, t) d a-\mu^{0} R(t),
\end{aligned}
$$


where $\eta(a)=\alpha(a)+\mu(a), \varrho(a)=\varepsilon(a)+\mu(a)$, and $\sigma(a)=$ $\gamma(a)+\mu(a)$, with boundary conditions

$$
\begin{aligned}
& v(0, t)=\nu S(t) \\
& e(0, t)=S(t) \int_{0}^{\infty}\left(K_{0}(a) i(a, t)\right. \\
& \left.+\int_{0}^{\infty} K\left(a, a^{\prime}\right) i\left(a^{\prime}, t\right) d a^{\prime}\right) d a \\
& i(0, t)=\int_{0}^{\infty} \varepsilon(a) e(a, t) d a .
\end{aligned}
$$

And initial conditions

$$
\begin{gathered}
S(0)=S_{0}>0, \\
v(a, 0)=v_{0}(a), \\
e(a, 0)=e_{0}(a)>0, \\
i(a, 0)=i_{0}(a)>0, \\
R(0)=R_{0}>0,
\end{gathered}
$$

where $S_{0}$ and $R_{0}$ are initial size of susceptible and removed individuals, respectively, and $v_{0}(a), e_{0}(a)$, and $i_{0}(a)$ are initial age-density of vaccinated, latent, and infective individuals, respectively. Moreover, $v_{0}, e_{0}$, and $i_{0}$ are Lebesgue integrable functions, and it is assumed that the recruitment of newly vaccinated individuals in the vaccinated compartment is done at age zero.

The meaning of parameters in (4)-(5) is given below:

$\Lambda$ : constant recruitment rate of susceptible individuals

$\nu$ : rate of vaccination of susceptible individuals

$\mu^{0}$ : natural mortality rate of individuals

$\alpha(a)$ : age-specific rate of waning vaccine-induced immunity

$\mu(a)$ : age-specific natural mortality rate

$\gamma(a)$ : age-specific removal rate

$\varepsilon(a)$ : age-specific rate moving from latent to infective

$K_{0}(a)$ : age-specific infection rate of susceptible individuals by infective individuals (of the same age-intracohort contagion)

$K\left(a, a^{\prime}\right)$ : probability that an infective individual of age $a^{\prime}$ will successfully infect a susceptible individual of age $a$, after contact

In the sequel, we make following assumptions on parameters in (4)-(5):

\section{A1}

(i) $\Lambda, \nu, \mu^{0}>0$ with $\nu<\mu^{0}$.

(ii) $\alpha, \eta, \varrho, \sigma, \gamma, \varepsilon, K_{0} \quad \in \quad \mathrm{L}_{+}^{\infty}(0, \infty)$ and $K \quad \epsilon$ $\mathrm{L}^{1}\left((0, \infty), \mathrm{L}_{+}^{\infty}(0, \infty)\right)$ with essential upper bounds $\bar{\alpha}, \bar{\eta}, \bar{\varrho}, \bar{\sigma}, \bar{\gamma}, \bar{\varepsilon}, \bar{K}_{0}$ and $\bar{K}(a, \cdot)$, respectively. (iii) $K_{0}(a), K\left(\cdot, a^{\prime}\right), \alpha(a), \gamma(a), \varepsilon(a)$ are Lipschitz continuous on $\mathbb{R}_{+}$with coefficients $M_{K_{0}}, M_{K}, M_{\alpha}, M_{\gamma}$, and $M_{\mathcal{\varepsilon}}$, respectively.

(iv) There exists $\tilde{\mu} \in\left(0, \mu^{0}\right]$ such that $\eta(a)-\alpha(a), \varrho(a)-$ $\varepsilon(a), \sigma(a)-\gamma(a)>\tilde{\mu}$.

A2. $R(\cdot)$ is a decreasing function of $t$ for any constant removal rate $\gamma^{0}$ such that $\gamma^{0} \geq \bar{\gamma}$.

A3. $\eta(a) \Lambda<(\eta(a)-\alpha(a))\left(1-\int_{0}^{\infty} \alpha(a) e^{-\int_{0}^{a} \eta(s) d s} d a\right)$, for every a.

This paper is composed sections, in addition to the introduction, which are structured as follows. In Section 2, we present some preliminary result on compactness property of the semiflow generated by (4)-(5) and we discuss its asymptotic smoothness property. The uniform persistence property of (4)-(5) is addressed in Section 4. Section 3 deals with the existence of equilibria and the formulation of the threshold parameter $\mathfrak{R}_{0}$ (the basic reproduction number). Local stability of equilibria for (4) is established in Section 5, while global stability of equilibria for (4) is examined in Section 6.

\section{Preliminaries}

We consider the Banach space

$$
\mathbb{X}=\mathbb{R} \times \mathrm{L}^{1}(0, \infty) \times \mathrm{L}^{1}(0, \infty) \times \mathrm{L}^{1}(0, \infty) \times \mathbb{R}
$$

endowed with the norm

$$
\|(x, \varphi, \psi, \phi, y)\|_{\mathbb{X}}=|x|+\|\varphi\|_{1}+\|\psi\|_{1}+\|\phi\|_{1}+|y|,
$$

where $\|\cdot\|_{1}=\|\cdot\|_{\mathrm{L}^{1}}$, for any $(x, \varphi, \psi, \phi, y) \in \mathbb{X}$. Let us denote by $\mathbb{X}_{+}$the positive cone of the Banach space $\mathbb{X}$ such that

$$
\mathbb{X}_{+}=\mathbb{R}_{+} \times \mathrm{L}_{+}^{1}(0, \infty) \times \mathrm{L}_{+}^{1}(0, \infty) \times \mathrm{L}_{+}^{1}(0, \infty) \times \mathbb{R}_{+} .
$$

For any initial value $\mathbf{X}_{0}=\left(S_{0}, v_{0}(\cdot), e_{0}(\cdot), i_{0}(\cdot), R_{0}\right) \in \mathbb{X}_{+}$ satisfying the conditions

$$
\begin{aligned}
& v_{0}(0)=\nu S_{0} \\
& \begin{aligned}
e_{0}(0) & =S_{0} \int_{0}^{\infty}\left(K_{0}(a) i_{0}(a)\right. \\
+ & \left.\int_{0}^{\infty} K\left(a, a^{\prime}\right) i_{0}\left(a^{\prime}\right) d a^{\prime}\right) d a \\
i_{0}(0) & =\int_{0}^{\infty} \varepsilon(a) e_{0}(a) d a
\end{aligned}
\end{aligned}
$$

system (4) is well-posed, under assumption A1, due to [21]. Thus, a continuous semiflow $\Phi: \mathbb{R}_{+} \times \mathbb{X}_{+} \longrightarrow \mathbb{X}_{+}$is obtained and it is defined by (4) such that

$$
\begin{aligned}
\Phi\left(t, \mathbf{X}_{0}\right) & =\Phi_{t}\left(\mathbf{X}_{0}\right) \\
= & (S(t), v(\cdot, t), e(\cdot, t), i(\cdot, t), R(t)), \\
& t \in \mathbb{R}_{+}, \mathbf{X}_{0} \in \mathbb{X}_{+} .
\end{aligned}
$$


Now, we introduce the functions

$$
\begin{aligned}
& \chi(a)=e^{-\int_{0}^{a} \eta(s) d s}, \\
& \vartheta(a)=e^{-\int_{0}^{a} \varrho(s) d s}, \\
& \zeta(a)=e^{-\int_{0}^{a} \sigma(s) d s} .
\end{aligned}
$$

By integrating the second, third, and fourth equations of (4) along the characteristic line $t-a=$ constant, respectively, we get

$$
\begin{aligned}
& v(a, t)= \begin{cases}v(0, t-a) \chi(a), & 0 \leq a \leq t, \\
v_{0}(a-t) \frac{\chi(a)}{\chi(a-t)}, & 0 \leq t \leq a,\end{cases} \\
& e(a, t)= \begin{cases}e(0, t-a) \vartheta(a), & 0 \leq a \leq t, \\
e_{0}(a-t) \frac{\vartheta(a)}{\vartheta(a-t)} & 0 \leq t \leq a,\end{cases} \\
& i(a, t)= \begin{cases}i(0, t-a) \zeta(a), & 0 \leq a \leq t, \\
i_{0}(a-t) \frac{\zeta(a)}{\zeta(a-t)} & 0 \leq t \leq a,\end{cases}
\end{aligned}
$$

where

$$
\begin{aligned}
& v(0, t-a)=\nu S(t-a) \\
& e(0, t-a)=S(t-a) \int_{0}^{\infty}\left(K_{0}(a) i(a, t-a)\right. \\
& \left.+\int_{0}^{\infty} K\left(a, a^{\prime}\right) i\left(a^{\prime}, t-a\right) d a^{\prime}\right) d a \\
& i(0, t-a)=\int_{0}^{\infty} \varepsilon(a) e(a, t-a) d a .
\end{aligned}
$$

Taking the norm of $\Phi_{t}\left(\mathbf{X}_{0}\right)$ and using the positiveness of the components of $\Phi_{t}\left(\mathbf{X}_{0}\right)$, we get

$$
\begin{aligned}
\left\|\Phi_{t}\left(\mathbf{X}_{0}\right)\right\|_{\mathbb{X}}= & S(t)+\|v(\cdot, t)\|_{1}+\|e(\cdot, t)\|_{1}+\|i(\cdot, t)\|_{1} \\
& +R(t) .
\end{aligned}
$$

Differentiating (15) with respect to $t$ leads to

$$
\begin{aligned}
\frac{\mathrm{d}}{\mathrm{d} t}\left\|\Phi_{t}\left(\mathbf{X}_{0}\right)\right\|_{\mathbb{X}}= & \frac{\mathrm{d} S(t)}{\mathrm{d} t}+\frac{\mathrm{d}}{\mathrm{d} t}\|v(\cdot, t)\|_{1}+\frac{\mathrm{d}}{\mathrm{d} t}\|e(\cdot, t)\|_{1} \\
& +\frac{\mathrm{d}}{\mathrm{d} t}\|i(\cdot, t)\|_{1}+\frac{\mathrm{d} R(t)}{\mathrm{d} t}
\end{aligned}
$$

Next, we seek for the estimates of each time-derivative on the right hand side (16). First, we have

$$
\begin{aligned}
& \frac{\mathrm{d}}{\mathrm{d} t}\|v(\cdot, t)\|_{1}=\frac{\mathrm{d}}{\mathrm{d} t}\left(\int_{0}^{t} v(0, t-a) \chi(a) d a\right. \\
& \left.+\int_{t}^{\infty} v_{0}(a-t) \frac{\chi(a)}{\chi(a-t)} d a\right)=\frac{\mathrm{d}}{\mathrm{d} t} \\
& \quad \cdot \int_{0}^{t} v(0, s) \chi(t-s) d s \\
& +\int_{0}^{\infty} \frac{v_{0}(\varsigma)}{\chi(\varsigma)} \frac{\mathrm{d}}{\mathrm{d} t} \chi(t+\varsigma) d \varsigma .
\end{aligned}
$$

Applying the Leibniz Integral Rule to the first integral in (17) yields

$$
\begin{aligned}
\frac{\mathrm{d}}{\mathrm{d} t}\|v(\cdot, t)\|_{1}= & \chi(0) v(0, t)+\int_{0}^{t} v(0, s) \frac{\mathrm{d}}{\mathrm{d} t} \chi(t-s) d s \\
& +\int_{0}^{\infty} \frac{v_{0}(\varsigma)}{\chi(\varsigma)} \frac{\mathrm{d}}{\mathrm{d} t} \chi(t+\varsigma) d \varsigma \\
= & v(0, t)-\int_{0}^{t} v(0, s) \eta(t-s) \chi(t-s) d s \\
& -\int_{0}^{\infty} v_{0}(\varsigma) \eta(t+\varsigma) \frac{\chi(t+\varsigma)}{\chi(\varsigma)} d \varsigma \\
= & \nu S(t)-\int_{0}^{\infty} \eta(a) v(a, t) d a .
\end{aligned}
$$

Likewise, we also have

$$
\frac{\mathrm{d}}{\mathrm{d} t}\|e(\cdot, t)\|_{1}=e(0, t)-\int_{0}^{\infty} \varrho(a) e(a, t) d a
$$

and

$$
\begin{aligned}
\frac{\mathrm{d}}{\mathrm{d} t}\|i(\cdot, t)\|_{1}= & \int_{0}^{\infty} \varepsilon(a) e(a, t) d a \\
& -\int_{0}^{\infty} \sigma(a) i(a, t) d a .
\end{aligned}
$$

Therefore, we get

$$
\begin{aligned}
\frac{\mathrm{d}}{\mathrm{d} t}\left(S(t)+\|v(\cdot, t)\|_{1}+\|e(\cdot, t)\|_{1}+\|i(\cdot, t)\|_{1}+R(t)\right) \\
=\Lambda-\mu^{0}(S(t)+R(t)) \\
\quad-\int_{0}^{\infty}(\eta(a)-\alpha(a)) v(a, t) d a \\
\quad-\int_{0}^{\infty}(\varrho(a)-\varepsilon(a)) v(a, t) d a \\
\quad-\int_{0}^{\infty}(\sigma(a)-\gamma(a)) i(a, t) d a .
\end{aligned}
$$


Using (iv) of A1, (21) yields

$$
\begin{aligned}
& \frac{\mathrm{d}}{\mathrm{d} t}\left(S(t)+\|v(\cdot, t)\|_{1}+\|e(\cdot, t)\|_{1}+\|i(\cdot, t)\|_{1}+R(t)\right) \\
& \quad \leq \Lambda-\tilde{\mu}\left(S(t)+\|v(\cdot, t)\|_{1}+\|e(\cdot, t)\|_{1}+\|i(\cdot, t)\|_{1}\right. \\
& \quad+R(t)),
\end{aligned}
$$

that is,

$$
\frac{\mathrm{d}}{\mathrm{d} t}\left\|\Phi_{t}\left(\mathbf{X}_{0}\right)\right\|_{\mathbb{X}} \leq \Lambda-\tilde{\mu}\left\|\Phi_{t}\left(\mathbf{X}_{0}\right)\right\|_{\mathbb{X}}
$$

Thus, we obtain

$$
\left\|\Phi_{t}\left(\mathbf{X}_{0}\right)\right\|_{\mathbb{X}} \leq \frac{\Lambda}{\widetilde{\mu}}-e^{-\widetilde{\mu} t}\left(\frac{\Lambda}{\tilde{\mu}}-\left\|\mathbf{X}_{0}\right\|_{\mathbb{X}}\right)
$$

where $\left\|\Phi_{0}\left(\mathbf{X}_{0}\right)\right\|_{\mathbb{X}}=\left\|\mathbf{X}_{0}\right\|_{\mathbb{X}}$

If we consider the state space $\Gamma$ of (4), defined by

$$
\Gamma=\left\{(x, \varphi, \psi, \phi, y) \in \mathbb{X}_{+}:\|(x, \varphi, \psi, \phi, y)\|_{\mathbb{X}} \leq \frac{\Lambda}{\widetilde{\mu}}\right\},
$$

we get

$$
\left\|\Phi_{t}\left(\mathbf{X}_{0}\right)\right\|_{\mathbb{X}} \leq \frac{\Lambda}{\tilde{\mu}}
$$

for any $t \geq 0$, whenever $\mathbf{X}_{0} \in \Gamma$. Moreover

$$
\limsup _{t \rightarrow \infty}\left\|\Phi_{t}\left(\mathbf{X}_{0}\right)\right\|_{\mathbb{X}} \leq \frac{\Lambda}{\widetilde{\mu}},
$$

for any $\mathbf{X}_{0} \in \mathbb{X}_{+}$.

Then, we state the following result.

Lemma 1. The set $\Gamma$ is positively invariant for $\Phi$; that is,

$$
\Phi_{t}\left(\mathbf{X}_{0}\right) \subset \Gamma, \quad \forall t \geq 0, \mathbf{X}_{0} \in \Gamma
$$

Moreover, $\Phi_{t}\left(\mathbf{X}_{0}\right)$ is point dissipative and the set $\Gamma$ attracts all points in $\mathbb{X}_{+}$.

As we aim to make use of the Lasalle's Invariance Principle, we are required to establish the relative compactness of the orbit $\left\{\Phi_{t}\left(\mathbf{X}_{0}\right): t \geq 0\right\}$ in $\mathbb{X}_{+}$due to the infinite dimensional Banach space $\mathbb{X}$. For this, we consider two operators $\Theta$ and $\Psi,\left(\Theta, \Psi: \mathbb{R}_{+} \times \mathbb{X}_{+} \longrightarrow \mathbb{X}_{+}\right)$, such that

$$
\begin{aligned}
\Theta\left(t, \mathbf{X}_{0}\right) & =\Theta_{t}\left(\mathbf{X}_{0}\right) \\
& =\left(0, \widetilde{\theta}_{v}(\cdot, a), \widetilde{\theta}_{e}(\cdot, a), \widetilde{\theta}_{i}(\cdot, a), 0\right) \\
\Psi\left(t, \mathbf{X}_{0}\right) & =\Psi_{t}\left(\mathbf{X}_{0}\right) \\
& =(S(t), \widetilde{v}(\cdot, a), \widetilde{e}(\cdot, a), \widetilde{i}(\cdot, a), R(t)),
\end{aligned}
$$

where

$$
\begin{aligned}
& \tilde{\theta}_{v}(a, t)= \begin{cases}0, & 0 \leq a \leq t, \\
v(a, t), & 0 \leq t \leq a,\end{cases} \\
& \widetilde{v}(a, t)= \begin{cases}v(a, t), & 0 \leq a \leq t, \\
0 & 0 \leq t \leq a,\end{cases}
\end{aligned}
$$$$
\tilde{\theta}_{e}(a, t)= \begin{cases}0, & 0 \leq a \leq t \\ e(a, t) & 0 \leq t \leq a,\end{cases}
$$$$
\tilde{e}(a, t)= \begin{cases}e(a, t), & 0 \leq a \leq t \\ 0 & 0 \leq t \leq a\end{cases}
$$

$$
\widetilde{\theta}_{i}(a, t)= \begin{cases}0, & 0 \leq a \leq t \\ i(a, t) & 0 \leq t \leq a\end{cases}
$$

$$
\tilde{i}(a, t)= \begin{cases}i(a, t), & 0 \leq a \leq t \\ 0 & 0 \leq t \leq a .\end{cases}
$$

Thus, $\Phi_{t}\left(\mathbf{X}_{0}\right)=\Theta_{t}\left(\mathbf{X}_{0}\right)+\Psi_{t}\left(\mathbf{X}_{0}\right)$, for any $t \geq 0$; and from the proof of [22, Proposition 3.13] and Lemma 1, we get to the following result.

Theorem 2. For $\mathbf{X}_{0} \in \Gamma$, the orbit $\left\{\Phi_{t}\left(\mathbf{X}_{0}\right): t \geq 0\right\}$ has a compact closure in $\mathbb{X}_{+}$if the following conditions are satisfied:

(i) There exists a function $\Delta: \mathbb{R}_{+} \times \mathbb{R}_{+} \longrightarrow \mathbb{R}_{+}$such that, for any $r>0, \lim _{t \rightarrow \infty} \Delta(t, r)=0$, and if $\mathbf{X}_{0} \in \Gamma$ with $\left\|\mathbf{X}_{0}\right\|_{\mathbb{X}} \leq r$, then $\left\|\Theta_{t}\left(\mathbf{X}_{0}\right)\right\|_{\mathbb{X}} \leq \Delta(t, r)$ for any $t \geq 0$.

(ii) For any $t \geq 0, \Psi_{t}(\cdot)$ maps any bounded sets of $\Gamma$ into a set with compact closure in $\mathbb{X}_{+}$.

For verifying conditions (i) and (ii) of Theorem 2, we need lemmas.

Lemma 3. For $r>0$, let $\Delta(t, r)=e^{-\widetilde{\mu} t} r$. Then $\lim _{t \rightarrow \infty} \Delta(t, r)=0$. Then (i) of Theorem 2 holds.

Proof. Clearly, we see that $\lim _{t \rightarrow \infty} \Delta(t, r)=0$. Making use of some equations in (13), we get

$$
\begin{aligned}
& \tilde{\theta}_{v}(a, t)= \begin{cases}0, & 0 \leq a \leq t, \\
v_{0}(a-t) \frac{\chi(a)}{\chi(a-t)} & 0 \leq t \leq a,\end{cases} \\
& \tilde{\theta}_{e}(a, t)= \begin{cases}0, & 0 \leq a \leq t, \\
e_{0}(a-t) \frac{\vartheta(a)}{\vartheta(a-t)} & 0 \leq t \leq a,\end{cases} \\
& \tilde{\theta}_{i}(a, t)= \begin{cases}0, & 0 \leq a \leq t, \\
i_{0}(a-t) \frac{\zeta(a)}{\zeta(a-t)} & 0 \leq t \leq a .\end{cases}
\end{aligned}
$$


Taking the initial condition $\mathbf{X}_{0} \in \Gamma$ such that $\left\|\mathbf{X}_{0}\right\|_{\mathbb{X}} \leq r$ and $t \geq 0$, we have

$$
\begin{aligned}
& \left\|\Theta_{t}\left(\mathbf{X}_{0}\right)\right\|_{\mathbb{X}}=|0|+\left\|\widetilde{\theta}_{v}(\cdot, t)\right\|_{1}+\left\|\widetilde{\theta}_{e}(\cdot, t)\right\|_{1} \\
& +\left\|\widetilde{\theta}_{i}(\cdot, t)\right\|_{1}+|0| \\
& =\int_{t}^{\infty}\left|v_{0}(a-t) \frac{\chi(a)}{\chi(a-t)}\right| d a \\
& +\int_{t}^{\infty}\left|e_{0}(a-t) \frac{\vartheta(a)}{\mathcal{Y}(a-t)}\right| d a \\
& +\int_{t}^{\infty}\left|i_{0}(a-t) \frac{\zeta(a)}{\zeta(a-t)}\right| d a \\
& =\int_{0}^{\infty}\left|v_{0}(s) \frac{\chi(s+t)}{\chi(s)}\right| d s \\
& +\int_{0}^{\infty}\left|e_{0}(s) \frac{\vartheta(s+t)}{\mathcal{\vartheta}(s)}\right| d s \\
& +\int_{0}^{\infty}\left|i_{0}(s) \frac{\zeta(s+t)}{\zeta(s)}\right| d s \\
& =\int_{0}^{\infty}\left|v_{0}(s) e^{-\int_{s}^{s+t} \eta(\tau) d \tau}\right| d s \\
& +\int_{0}^{\infty}\left|e_{0}(s) e^{-\int_{s}^{s+t} e(\tau) d \tau \mid}\right| d s \\
& +\int_{0}^{\infty}\left|i_{0}(s) e^{-\int_{s}^{s+t} \sigma(\tau) d \tau}\right| d s \\
& \leq e^{-\widetilde{\mu} t}\left(\left\|v_{0}\right\|_{1}+\left\|e_{0}\right\|_{1}+\left\|i_{0}\right\|_{1}\right) \\
& \leq e^{-\widetilde{\mu} t}\left\|\mathbf{X}_{0}\right\|_{\mathbb{X}} \leq e^{-\widetilde{\mu} t} r=\Lambda(t, r) \text {. }
\end{aligned}
$$

Lemma 4. For $t \geq 0, \Psi_{t}(\cdot)$ maps any bounded set of $\Gamma$ into a set with a compact closure in $\mathbb{X}_{+}$.

Proof. Since $S(t)$ and $R(t)$ remain in the compact set $[0, \Lambda / \widetilde{\mu}]$ by Lemma 1 , it is sufficient to show that $\widetilde{v}(a, t), \widetilde{e}(a, t)$, and $\widetilde{i}(a, t)$ remain in a precompact subset of $\mathrm{L}_{+}^{1}(0, \infty)$, which does not depend on the initial data $\mathbf{X}_{0} \in \Gamma$. To achieve this, the following conditions (see [23, Theorem B.2]) must be satisfied for $\widetilde{v}(a, t), \widetilde{e}(a, t)$, and $\widetilde{i}(a, t)$ :

(i) The supremum of $\|\widetilde{z}(\cdot, t)\|_{1}$ with respect to $\mathbf{X}_{0} \in \Gamma$ is finite;

(ii) $\lim _{h \rightarrow \infty} \int_{h}^{\infty} \tilde{z}(a, t) d a=0$ uniformly with respect to $\mathbf{X}_{0} \in \Gamma$;

(iii) $\lim _{h \rightarrow 0^{+}} \int_{h}^{\infty}|\widetilde{z}(a+h, t)-\widetilde{z}(a, t)| d a=0$ uniformly with respect to $\mathbf{X}_{0} \in \Gamma$;

(iv) $\lim _{h \rightarrow 0^{+}} \int_{0}^{h} \widetilde{z}(a, t) d a=0$ uniformly with respect to $\mathbf{X}_{0} \in \Gamma$, where $\widetilde{z} \in\{\widetilde{v}, \widetilde{e}, \widetilde{i}\}$. It follows from (13) and (30) that

$$
\widetilde{e}(a, t)= \begin{cases}e_{0}(t-a) \vartheta(a), & 0 \leq a \leq t, \\ 0, & 0 \leq t \leq a,\end{cases}
$$

and hence, using Lemma 1 , we get

$$
0 \leq \widetilde{v}(a, t) \leq \nu \frac{\Lambda}{\tilde{\mu}} e^{-\widetilde{\mu} a},
$$

and hence, (i), (ii), and (iv) follow. To establish (iii), we take a sufficiently small $h$ such that $h \in(0, t)$ and we show that

$$
\begin{aligned}
& \int_{0}^{\infty}|\widetilde{v}(a+h, t)-\widetilde{v}(a, t)| d a \\
& =\int_{t-h}^{t}|0-\nu S(t-a) \chi(a)| d a \\
& +v \int_{0}^{t-h}|S(t-a-h) \chi(a+h)-S(a, t) \chi(a)| d a \\
& \leq \nu \frac{\Lambda}{\tilde{\mu}} h+\nu \int_{0}^{t-h} S(t-a-h)(\chi(a)-\chi(a+h)) d a \\
& +v \int_{0}^{t-h} \chi(a)|S(t-a-h)-S(a, t)| d a \\
& \leq \nu \frac{\Lambda}{\tilde{\mu}} h+\nu \frac{\Lambda}{\widetilde{\mu}}\left(\int_{0}^{t-h} \chi(a) d a-\int_{0}^{t-h} \chi(a+h) d a\right) \\
& +v \int_{0}^{t-h} \chi(a)|S(t-a-h)-S(a, t)| d a \\
& \leq \nu \frac{\Lambda}{\widetilde{\mu}} h+\nu \frac{\Lambda}{\widetilde{\mu}}\left(\int_{0}^{t} \chi(a) d a+\int_{t}^{h} \chi(a) d a\right) \\
& +v \int_{0}^{t-h} \chi(a)|S(t-a-h)-S(a, t)| d a \\
& \leq v\left(2 \Lambda+l_{S}\right) \frac{h}{\widetilde{\mu}} .
\end{aligned}
$$

Indeed, $\chi(a)$ is a nondecreasing function of $a$ such that $0 \leq$ $\chi(a) \leq 1$ satisfying

$$
\begin{aligned}
\int_{0}^{t-h} & |\chi(a+h)-\chi(a)| d a \\
& =\int_{0}^{t-h}(\chi(a)-\chi(a+h)) d a \\
& =\int_{0}^{t-h} \chi(a) d a-\int_{0}^{t-h} \chi(a+h) d a \\
& =\int_{0}^{t-h} \chi(a) d a-\int_{h}^{t} \chi(a) d a \\
& =\int_{0}^{t-h} \chi(a) d a-\int_{h}^{t-h} \chi(a) d a-\int_{t-h}^{t} \chi(a) d a \\
& =\int_{0}^{h} \chi(a) d a-\int_{t-h}^{t} \chi(a) d a \leq \int_{0}^{h} \chi(a) d a \leq h .
\end{aligned}
$$


On the other hand, the Lipschitz continuity of $S(\cdot)$ is obtained from the first equation of (4), using the boundedness of the solution of (4) (see Lemma 1); that is, there exists $l_{S}>0$ such that $\left|S\left(t_{1}\right)-S\left(t_{2}\right)\right| \leq l_{S}\left|t_{1}-t_{2}\right|$ for any $t_{1}, t_{2} \geq 0$.

Since $\nu\left(2 \Lambda+l_{S}\right) h / \widetilde{\mu}$ does not depend on the initial data $\mathbf{X}_{0} \in \Gamma$ and $\nu\left(2 \Lambda+l_{S}\right) h / \tilde{\mu} \longrightarrow 0$ as $h \longrightarrow 0^{+}$, it follows from (35) that (iii) is satisfied.

Therefore, From Lemma 1 and Theorem 2, the existence result of global attractors (see [24]) follows.

Theorem 5. The semiflow $\left\{\Phi_{t}\left(\mathbf{X}_{0}\right): t \geq 0\right\}$ has a global attractor in $\mathbb{X}_{+}$, which attracts any bounded subset of $\mathbb{X}_{+}$.

\section{Equilibria and Basic Reproduction Number}

System (4) has a unique disease-free equilibrium $E^{0}=$ $\left(S^{0}, v^{0}(a), e^{0}(a), i^{0}, R^{0}\right)$, where

$$
\begin{aligned}
S^{0} & =\frac{\Lambda}{\mu^{0}+\nu\left(1-\int_{0}^{\infty} \alpha(a) e^{-\int_{0}^{a} \eta(s) d s} d a\right)}, \\
v^{0}(a) & =\nu S^{0} e^{-\int_{0}^{a} \eta(s) d s}, \\
e^{0}(a) & =i^{0}(a)=0, \\
R^{0} & =0 .
\end{aligned}
$$

Apart from $E^{0}$, system (4) could also have an endemic equilibrium. We suppose that there exists an endemic equilibrium for system (4) denoted by $E^{*}=\left(S^{*}, v^{*}, e^{*}, i^{*}, R^{*}\right)$. Therefore,

$$
\begin{aligned}
0= & \Lambda-\left(\nu+\mu^{0}\right) S^{*}+\int_{0}^{\infty} \alpha(a) v^{*}(a) d a \\
& -S^{*} \int_{0}^{\infty}\left(K_{0}(a) i^{*}(a)\right. \\
& \left.+\int_{0}^{\infty} K\left(a, a^{\prime}\right) i^{*}\left(a^{\prime}\right) d a^{\prime}\right) d a \\
0= & -\frac{\mathrm{d}}{\mathrm{d} a} v^{*}(a)-\eta(a) v^{*}(a) \\
0= & -\frac{\mathrm{d}}{\mathrm{d} a} e^{*}(a)-\varrho(a) e^{*}(a) \\
0= & -\frac{\mathrm{d}}{\mathrm{d} a} i^{*}(a)-\sigma(a) i^{*}(a) \\
0= & \int_{0}^{\infty} \gamma(a) i^{*}(a) d a-\mu^{0} R^{*} .
\end{aligned}
$$

are satisfied. In addition, $E^{*}$ also satisfies (5), i.e.,

$$
\begin{aligned}
& \begin{array}{l}
v^{*}(0)=\nu S^{*} \\
e^{*}(0)=S^{*} \int_{0}^{\infty}\left(K_{0}(a) i^{*}(a)\right. \\
\left.+\int_{0}^{\infty} K\left(a, a^{\prime}\right) i^{*}\left(a^{\prime}\right) d a^{\prime}\right) d a \\
i^{*}(0)=\int_{0}^{\infty} \varepsilon(a) e^{*}(a) d a .
\end{array}
\end{aligned}
$$

The second equation of (38) and the first equation of (39) give

$$
v^{*}(a)=\nu S^{*} e^{-\int_{0}^{a} \eta(s) d s} .
$$

It follows from the third equation of (38) that

$$
e^{*}(a)=e^{*}(0) e^{-\int_{0}^{a} \varrho(s) d s} .
$$

Equations (5) and (41) together with the fourth equation of (38) yield

$$
i^{*}(a)=e^{*}(0)\left(e^{-\int_{0}^{a} \sigma(s) d s} \int_{0}^{\infty} \mathcal{\varepsilon}(a) e^{-\int_{0}^{a} \varrho(s) d s} d a\right) .
$$

We introduce parameters $\mathfrak{R}_{0}, L, J$, and $P$ such that

$$
\begin{aligned}
\mathfrak{R}_{0} & =S^{0} L \int_{0}^{\infty}\left(K_{0}(a) e^{-\int_{0}^{a} \sigma(s) d s}\right. \\
& \left.+\int_{0}^{\infty} K\left(a, a^{\prime}\right) e^{-\int_{0}^{a^{\prime}} \sigma(s) d s} d a^{\prime}\right) d a \\
L & =\int_{0}^{\infty} \varepsilon(a) e^{-\int_{0}^{a} \varrho(s) d s} d a \\
J & =\int_{0}^{\infty} \gamma(a) e^{-\int_{0}^{a} \sigma(s) d s} d a \\
P & =\int_{0}^{\infty} \alpha(a) e^{-\int_{0}^{a} \eta(s) d s} d a .
\end{aligned}
$$

By substituting (42) into the second equation of (39), we have

$$
S^{*}=\frac{S^{0}}{\mathfrak{R}_{0}}
$$

And hence,

$$
v^{*}(a)=\frac{v^{0}(a)}{\mathfrak{R}_{0}} .
$$

Then, substituting (37), (40), (42), and (44) into the first equation of (38) yields

$$
e^{*}(0)=\Lambda\left(1-\frac{1}{\mathfrak{R}_{0}}\right) .
$$


Therefore, it easily follows that

$$
\begin{aligned}
e^{*}(a) & =\Lambda\left(1-\frac{1}{\mathfrak{R}_{0}}\right) \vartheta(a) \\
i^{*}(a) & =\Lambda\left(1-\frac{1}{\mathfrak{R}_{0}}\right) L \zeta(a) \\
R^{*} & =\frac{\Lambda}{\mu^{0}}\left(1-\frac{1}{\mathfrak{R}_{0}}\right) L J .
\end{aligned}
$$

A threshold condition is derived from the existence condition for the endemic equilibrium $E^{*}$ such that $\mathfrak{R}_{0}>1$. Thus, the parameter $\mathfrak{R}_{0}$, given by the first equation of (43), can be called the basic reproduction number of system (4). Moreover, $\mathfrak{R}_{0}$ can also be expressed as

$$
\mathfrak{R}_{0}=\mathfrak{R}_{\text {intra }}+\mathfrak{R}_{\text {inter }},
$$

where

$$
\begin{aligned}
& \mathfrak{R}_{\text {intra }}=\frac{\Lambda L}{\mu^{0}+\nu(1-P)} \int_{0}^{\infty} K_{0}(a) \zeta(a) d a, \\
& \boldsymbol{R}_{\text {inter }} \\
& \quad=\frac{\Lambda L}{\mu^{0}+v(1-P)} \int_{0}^{\infty}\left(\int_{0}^{\infty} K\left(a, a^{\prime}\right) \zeta\left(a^{\prime}\right) d a^{\prime}\right) d a .
\end{aligned}
$$

$\mathfrak{R}_{\text {intra }}$ and $\mathfrak{R}_{\text {inter }}$ can be understood, respectively, as the basic reproduction numbers for the corresponding model with purely intracohort infection mechanism (i.e., situation in which individuals can only be infected by their age-mates) and for the corresponding model with purely intercohort infection mechanism (i.e., situation in which individuals can be infected by those of any age).

Therefore, we state the following.

Theorem 6. If $\mathfrak{R}_{0} \leq 1$, then system (4) has only a diseasefree equilibrium $E^{0}$, while if $\mathfrak{R}_{0}>1$, then system (4) also has an endemic equilibrium $E^{*}$ in addition to the disease-free equilibrium $E^{0}$.

\section{Uniform Persistence}

This section is devoted to the uniform persistence of system (4) under the condition $\mathfrak{R}_{0}>1$. For this, we introduce a function $\rho: \mathbb{X}_{+} \longrightarrow \mathbb{R}_{+}$defined by

$$
\begin{aligned}
& \rho(x, \varsigma, \omega, v, y) \\
& =x \int_{0}^{\infty}\left(K_{0}(a) v(a)+\int_{0}^{\infty} K\left(a, a^{\prime}\right) v\left(a^{\prime}\right) d a^{\prime}\right) d a,
\end{aligned}
$$

where $(x, \varsigma, \omega, v, y) \in \mathbb{X}_{+}$. Furthermore, we consider the set $\mathbb{X}_{0}$ defined by

$$
\begin{aligned}
& \mathbb{X}_{0} \\
& \quad=\left\{\mathbf{X}_{0} \in \mathbb{X}_{+}: \rho\left(\Phi_{t_{0}}\left(\mathbf{X}_{0}\right)\right)>0 \text { for some } t_{0} \in \mathbb{R}_{+}\right\}
\end{aligned}
$$

such that $\Phi_{t}\left(\mathbf{X}_{0}\right) \longrightarrow E^{0}$ as $t \longrightarrow \infty$ whenever $\mathbf{X}_{0} \in \mathbb{X}_{+} \backslash \mathbb{X}_{0}$.
Definition 7 (see [23, p. 61]). System (4) is uniformly weakly $\rho$-persistent (respectively, uniformly strongly $\rho$-persistent) if there exists a positive $\epsilon^{*}$, independent of initial conditions, such that

$$
\begin{aligned}
& \limsup _{t \rightarrow \infty} \rho\left(\Phi_{t}\left(\mathbf{X}_{0}\right)\right) \\
& \quad>\epsilon^{*}\left(\text { respectively, } \liminf _{t \rightarrow \infty} \rho\left(\Phi_{t}\left(\mathbf{X}_{0}\right)\right)>\epsilon^{*}\right)
\end{aligned}
$$

for $\mathbf{X}_{0} \in \mathbb{X}_{+}$.

Theorem 8. If $\mathfrak{R}_{0}>1$, then system (4) is uniformly weakly $\rho$-persistent.

Proof. We assume that, for any $\epsilon^{*}>0$, we can find $\mathbf{X}_{0}^{\epsilon^{*}} \in \mathbb{X}_{+}$ such that

$$
\limsup _{t \rightarrow \infty} \rho\left(\Phi_{t}\left(\mathbf{X}_{0}^{\epsilon^{*}}\right)\right) \leq \epsilon^{*}
$$

Since $\mathfrak{R}_{0}>1$, then we can find a small enough $\epsilon_{0}^{*}>0$ such that

$$
\begin{aligned}
1< & \left(\frac{\Lambda-\epsilon_{0}^{*}}{\nu+\mu_{0}}-\epsilon_{0}^{*}\right)\left(\int_{0}^{\infty} \varepsilon(t) \vartheta(t) d t\right) \\
& \cdot \int_{0}^{\infty}\left(K_{0}(a) \zeta(a)+\int_{0}^{\infty} K\left(a, a^{\prime}\right) \zeta\left(a^{\prime}\right) d a^{\prime}\right) d a .
\end{aligned}
$$

In particular, we can find $\mathbf{X}_{0}^{\epsilon_{0}^{*} / 2} \in \mathbb{X}_{+}$such that

$$
\limsup _{t \rightarrow \infty} \rho\left(\Phi_{t}\left(\mathbf{X}_{0}^{\epsilon_{0}^{*} / 2}\right)\right) \leq \frac{\epsilon_{0}^{*}}{2} .
$$

We can assume that, for any $t \geq 0, \rho\left(\Phi_{t}\left(\mathbf{X}_{0}^{\epsilon_{0}^{*} / 2}\right)\right) \leq \epsilon_{0}^{*}$. It follows from the first equation of (4) that

$$
\begin{aligned}
\frac{\mathrm{d}}{\mathrm{d} t} S(t) \geq & \Lambda-\epsilon_{0}^{*}-\left(\nu+\mu^{0}\right) S(t)+\int_{0}^{t} \alpha(a) v(a, t) d a \\
= & \Lambda-\epsilon_{0}^{*}-\left(\nu+\mu^{0}\right) S(t) \\
& +\int_{0}^{t} \alpha(a) \zeta(a) v(0, t-a) d a \\
\geq & \Lambda-\epsilon_{0}^{*}-\left(\nu+\mu^{0}\right) S(t) \\
& +v \int_{0}^{t} \alpha(a) \chi(a) S(t-a) d a .
\end{aligned}
$$

We apply the Laplace transform $\mathscr{L}$ to the above inequality and we obtain

$$
\begin{aligned}
\lambda \mathscr{L}\{S(t)\}-S_{0} \geq & \frac{\Lambda-\epsilon_{0}^{*}}{\lambda}-\left(\nu+\mu^{0}\right) \mathscr{L}\{S(t)\} \\
& +\nu \mathscr{L}\{\alpha(t) \chi(t)\} \mathscr{L}\{S(t)\} .
\end{aligned}
$$


It follows that

$$
\begin{aligned}
\mathscr{L}\{S(t)\} & \geq \frac{S_{0} \lambda+\Lambda-\epsilon_{0}^{*}}{\lambda\left(\lambda+\mu^{0}+\nu(1-\mathscr{L}\{\alpha(t) \chi(t)\})\right)} \\
& \geq \frac{S_{0} \lambda+\Lambda-\epsilon_{0}^{*}}{\lambda\left(\lambda+\mu^{0}+\nu\right)} \\
& =\frac{\Lambda-\epsilon_{0}^{*}}{\nu+\mu^{0}} \cdot \frac{1}{\lambda}+\left(S_{0}-\frac{\Lambda-\epsilon_{0}^{*}}{\nu+\mu^{0}}\right) \frac{1}{\lambda+\nu+\mu^{0}}
\end{aligned}
$$

And hence,

$$
\begin{aligned}
S(t) \geq & \frac{\Lambda-\epsilon_{0}^{*}}{\nu+\mu^{0}} \mathscr{L}^{-1}\left\{\frac{1}{\lambda}\right\} \\
& +\left(S_{0}-\frac{\Lambda-\epsilon_{0}^{*}}{\nu+\mu^{0}}\right) \mathscr{L}^{-1}\left\{\frac{1}{\lambda+v+\mu^{0}}\right\} \\
= & \frac{\Lambda-\epsilon_{0}^{*}}{\nu+\mu^{0}}+e^{-\left(\nu+\mu^{0}\right) t}\left(S_{0}-\frac{\Lambda-\epsilon_{0}^{*}}{\nu+\mu^{0}}\right),
\end{aligned}
$$

for any $t \geq 0$.

This yields $\lim \sup _{t \rightarrow \infty} S(t) \geq\left(\Lambda-\epsilon_{0}\right) /\left(\nu+\mu^{0}\right)$. We can assume that, for any $t \geq 0$,

$$
S(t) \geq \frac{\Lambda-\epsilon_{0}^{*}}{\nu+\mu^{0}}-\epsilon_{0}^{*} .
$$

Now, we consider the boundary condition defined by the second equation of (5) and we get

$$
\begin{aligned}
& e(0, t) \geq S(t) \int_{0}^{t}\left(K_{0}(a) i(a, t)+\int_{0}^{t} K\left(a, a^{\prime}\right)\right. \\
& \left.\cdot i\left(a^{\prime}, t\right) d a^{\prime}\right) d a \geq\left(\frac{\Lambda-\epsilon_{0}^{*}}{\nu+\mu^{0}}-\epsilon_{0}^{*}\right)\left(\int_{0}^{t} K_{0}(a)\right. \\
& \left.\cdot i(a, t) d a+\int_{0}^{t} \int_{0}^{t} K\left(a, a^{\prime}\right) i\left(a^{\prime}, t\right) d a^{\prime} d a\right) \\
& \geq\left(\frac{\Lambda-\epsilon_{0}^{*}}{\nu+\mu^{0}}-\epsilon_{0}^{*}\right)\left(\int_{0}^{t} K_{0}(a) \zeta(a) i(0, t-a) d a\right. \\
& \left.+\int_{0}^{t} \int_{0}^{t} K\left(a, a^{\prime}\right) \zeta\left(a^{\prime}\right) i\left(0, t-a^{\prime}\right) d a^{\prime} d a\right) \geq\left(\frac{\Lambda-\epsilon_{0}^{*}}{\nu+\mu^{0}}\right. \\
& \left.-\epsilon_{0}^{*}\right)\left(\int_{0}^{t} K_{0}(a) \zeta(a)\right. \\
& \cdot\left(\int_{0}^{t-a} \varepsilon(b) \vartheta(b) e(0, t-a-b) d b\right) d a \\
& +\int_{0}^{t} \int_{0}^{t} K\left(a, a^{\prime}\right) \zeta\left(a^{\prime}\right) \\
& \left..\left(\int_{0}^{t-a^{\prime}} \varepsilon\left(b^{\prime}\right) \vartheta\left(b^{\prime}\right) e\left(0, t-a^{\prime}-b^{\prime}\right) d b^{\prime}\right) d a^{\prime} d a\right) .
\end{aligned}
$$

We apply the Laplace Transform $\mathscr{L}$ to the above inequality so that

$$
\begin{aligned}
& \mathscr{L}\{e(0, t)\} \geq\left(\frac{\Lambda-\epsilon_{0}^{*}}{\nu+\mu^{0}}-\epsilon_{0}^{*}\right) \\
& \cdot\left(\mathscr{L}\left\{K_{0}(t) \zeta(t)\right\}+\frac{1}{\lambda} \mathscr{L}\{K(\cdot, t) \zeta(t)\}\right) \\
& \cdot \mathscr{L}\{\varepsilon(t) \vartheta(t)\} \mathscr{L}\{e(0, t)\} .
\end{aligned}
$$

Dividing the above inequality by $\mathscr{L}\{e(0, t)\}$ yields

$$
\begin{aligned}
1 \geq & \left(\frac{\Lambda-\epsilon_{0}^{*}}{\nu+\mu^{0}}-\epsilon_{0}^{*}\right)\left(\mathscr{L}\left\{K_{0}(t) \zeta(t)\right\}+\frac{1}{\lambda}\right. \\
& . \mathscr{L}\{K(\cdot, t) \zeta(t)\}) \mathscr{L}\{\varepsilon(t) \vartheta(t)\} \geq\left(\frac{\Lambda-\epsilon_{0}^{*}}{\nu+\mu^{0}}\right. \\
& \left.-\epsilon_{0}^{*}\right)\left(\int_{0}^{\infty} e^{-\lambda t} \varepsilon(t) \vartheta(t) d t\right) \\
& \times\left(\int_{0}^{\infty} e^{-\lambda t} K_{0}(t) \zeta(t) d t\right. \\
& \left.+\int_{0}^{\infty} e^{-\lambda t}\left(\int_{0}^{t} K(t, s) \zeta(s) d s\right) d t\right)=\left(\frac{\Lambda-\epsilon_{0}^{*}}{\nu+\mu^{0}}\right. \\
& \left.-\epsilon_{0}^{*}\right)\left(\int_{0}^{\infty} e^{-\lambda t} \varepsilon(t) \vartheta(t) d t\right) \\
& \times\left(\int_{0}^{\infty} e^{-\lambda t} K_{0}(t) \zeta(t) d t\right. \\
& \left.+\int_{0}^{\infty} \int_{0}^{t} e^{-\lambda t} K(t, s) \zeta(s) d s d t\right)=\left(\frac{\Lambda-\epsilon_{0}^{*}}{\nu+\mu^{0}}-\epsilon_{0}^{*}\right) \\
& +\left(\int_{0}^{\infty} e^{-\lambda t} \varepsilon(t) \vartheta(t) d t\right) \times \int_{0}^{\infty}\left(e^{-\lambda t} K_{0}(t) \zeta(t)\right. \\
& \left.+\int_{0}^{t} e^{-\lambda t} K(t, s) \zeta(s) d s\right) d t .
\end{aligned}
$$

First, taking the limit inferior as $t \longrightarrow \infty$ on both sides of the above inequality, we obtain

$$
\begin{aligned}
1 \geq & \left(\frac{\Lambda-\epsilon_{0}^{*}}{\nu+\mu^{0}}-\epsilon_{0}^{*}\right)\left(\int_{0}^{\infty} e^{-\lambda t} \varepsilon(t) \vartheta(t) d t\right) \\
& \times \int_{0}^{\infty}\left(e^{-\lambda t} K_{0}(t) \zeta(t)\right. \\
& \left.+\liminf _{t \rightarrow \infty}^{t} \int_{0}^{t} e^{-\lambda t} K(t, s) \zeta(s) d s\right) d t \\
& =\left(\frac{\Lambda-\epsilon_{0}^{*}}{\nu+\mu^{0}}-\epsilon_{0}^{*}\right)\left(\int_{0}^{\infty} e^{-\lambda t} \varepsilon(t) \vartheta(t) d t\right) \\
& \times \int_{0}^{\infty}\left(e^{-\lambda t} K_{0}(t) \zeta(t)\right. \\
& \left.+\int_{0}^{\infty} e^{-\lambda t} K(t, s) \zeta(s) d s\right) d t .
\end{aligned}
$$


Next, take the limit as $\lambda \longrightarrow 0$ on both sides of the above inequality, we get

$$
\begin{aligned}
1 \geq & \left(\frac{\Lambda-\epsilon_{0}^{*}}{\nu+\mu^{0}}-\epsilon_{0}^{*}\right)\left(\int_{0}^{\infty} \varepsilon(t) \vartheta(t) d t\right) \\
& \times \int_{0}^{\infty}\left(K_{0}(t) \zeta(t)+\int_{0}^{\infty} K(t, s) \zeta(s) d s\right) d t,
\end{aligned}
$$

which contradicts the inequality given in (54).

Combining the results from Theorems 5 and 8 with [25, Theorem 3.2] leads to the uniform (strong) $\rho$-persistence as follows.

Theorem 9. If $\mathfrak{R}_{0}>1$, then the semiflow $\Phi$ is uniformly (strongly) $\rho$-persistent.

Definition 10. A total trajectory of a continuous semiflow $\Phi$, defined by (11), is a function $\mathbf{X}: \mathbb{R} \longrightarrow \mathbb{X}_{+}$such that $\Phi_{t}(\mathbf{X}(r))=\mathbf{X}(t+r)$ for any $(t, r) \in \mathbb{R}_{+} \times \mathbb{R}$.

Note that a global attractor will only contain points with total trajectories through them as it needs to be invariant. So, the $\alpha$-limit point of a total trajectory $\mathbf{X}$, passing through $\mathbf{X}(0)=\mathbf{X}_{0}$, is given by

$$
\alpha\left(\mathbf{X}_{0}\right)=\bigcap_{t \geq 0} \overline{\bigcup_{s \geq t} \mathbf{X}(s)} .
$$

A total trajectory $\mathbf{X}(t)=(S(t), v(\cdot, t), e(\cdot, t), i(\cdot, t), R(t))$ satisfies

$$
\begin{array}{ll}
v(a, r)=v S(r-a) \chi(a), & (a, r) \in \mathbb{R}_{+} \times \mathbb{R}, \\
e(a, r)=e(0, r-a) \vartheta(a), & (a, r) \in \mathbb{R}_{+} \times \mathbb{R}, \\
i(a, r)=i(0, r-a) \zeta(a), & (a, r) \in \mathbb{R}_{+} \times \mathbb{R} .
\end{array}
$$

Corollary 11. Let $\mathscr{A}$ and $\mathbf{X}(t)$ be, respectively, a global attractor of $\Phi$ in $\mathbb{X}_{+}$and a total trajectory of $\Phi$ in $\mathscr{A} \cap \mathbb{X}_{+}$. If $\mathfrak{R}_{0}>1$, then there exists $\epsilon>0$ such that

$$
S(t), v(0, t), e(0, t), i(0, t), R(t) \geq \epsilon,
$$

$$
\text { for any } t \geq 0 \text {. }
$$

Proof. We consider the boundary condition given by the second equation of (5). Using (25) and (ii) of A1, we get

$$
\begin{aligned}
e(0, t) & \leq 4\|i(t)\|_{1} S(t) \max \left\{\bar{K}_{0},\|\bar{K}\|_{1}\right\} \\
& \leq 4 \frac{\Lambda^{2}}{\widetilde{\mu}^{2}} \max \left\{\bar{K}_{0},\|\bar{K}\|_{1}\right\}=: \overline{\mathscr{K}} .
\end{aligned}
$$

From the first equation of (4), we have

$$
\begin{aligned}
& \frac{\mathrm{d}}{\mathrm{d} t} S(t) \geq \Lambda-\left(\nu-\mu^{0}\right) S(t)-S(t) \\
& \quad \cdot \int_{0}^{\infty}\left(K_{0}(a) \zeta(a) i(0, t-a)\right. \\
& \left.\quad+\int_{0}^{\infty} K\left(a, a^{\prime}\right) \zeta(a) i\left(0, t-a^{\prime}\right) d a^{\prime}\right) d a \\
& \geq \Lambda-\left(\nu-\mu^{0}\right) S(t)-L \overline{\mathscr{K}} S(t) \int_{0}^{\infty}\left(K_{0}(a) \zeta(a)\right. \\
& \left.\quad+\int_{0}^{\infty} K\left(a, a^{\prime}\right) \zeta(a) d a^{\prime}\right) d a \geq \Lambda-\left(\nu+\mu^{0}\right. \\
& \left.\quad+\frac{\overline{\mathscr{K}}}{S^{0}} \Re_{0}\right) S(t) ;
\end{aligned}
$$

that is,

$$
\frac{\mathrm{d}}{\mathrm{d} t} S(t) \geq \Lambda-\left(\nu+\mu^{0}+\frac{\overline{\mathscr{K}}}{S^{0}} \Re_{0}\right) S(t) .
$$

This yields

$$
\begin{aligned}
S(t) & \\
\geq & \frac{\Lambda S^{0}}{\left(\nu+\mu^{0}\right) S^{0}+\overline{\mathscr{K}} \Re_{0}} \\
& +e^{-\left(\nu+\mu^{0}+\left(\overline{\mathscr{K}} / S^{0}\right) \Re_{0}\right) t}\left(S_{0}-\frac{\Lambda S^{0}}{\left(\nu+\mu^{0}\right) S^{0}+\overline{\mathscr{K}} \mathfrak{R}_{0}}\right) .
\end{aligned}
$$

Taking the limit inferior as $t \longrightarrow \infty$ in (72) leads to

$$
\liminf _{t \longrightarrow \infty} S(t) \geq \frac{\Lambda S^{0}}{\left(\nu+\mu^{0}\right) S^{0}+\overline{\mathscr{K}} \Re_{0}}=: \epsilon_{1} .
$$

Therefore, $S(t) \geq \epsilon_{1}$. It follows that $v(0, t) \geq \epsilon_{1} v=: \epsilon_{2}$.

Now, we consider again the boundary condition given by the second equation of (5). It is easy to see that

$$
e(0, t)=\rho\left(\Phi_{t}\left(\mathbf{X}_{0}\right)\right)=\rho(\mathbf{X}(t))
$$

And hence,

$$
e(0, t) \geq \liminf _{t \rightarrow \infty} \rho(\mathbf{X}(t))
$$

It follows from Theorem 9 and Definition 7 that $e(0, t) \geq \epsilon^{*}=$ : $\epsilon_{3}$.

Further, considering the boundary condition given by the third equation of (5), we obtain

$$
\begin{aligned}
i(0, t) & =\int_{0}^{\infty} \varepsilon(a) \vartheta(a) e(0, t-a) d a \\
& \geq \epsilon_{3} \int_{0}^{\infty} \varepsilon(a) \vartheta(a) d a=: \epsilon_{4} .
\end{aligned}
$$


Finally, the fifth equation of (4) leads to

$$
\frac{\mathrm{d}}{\mathrm{d} t} R(t) \geq \epsilon_{4} \int_{0}^{\infty} \gamma(a) \zeta(a) d a-\mu^{0} R(t)
$$

And hence,

$$
\begin{aligned}
R(t) \geq & \frac{\epsilon_{4}}{\mu^{0}} \int_{0}^{\infty} \gamma(a) \zeta(a) d a \\
& +e^{-\mu^{0} t}\left(R_{0}-\frac{\epsilon_{4}}{\mu^{0}} \int_{0}^{\infty} \gamma(a) \zeta(a) d a\right) .
\end{aligned}
$$

Taking the limit inferior as $t \longrightarrow \infty$ in (78), we obtain

$$
\liminf _{t \rightarrow \infty} R(t) \geq \frac{\epsilon_{4}}{\mu^{0}} \int_{0}^{\infty} \gamma(a) \zeta(a) d a=: \epsilon_{5} .
$$

And therefore $R(t) \geq \epsilon_{5}$. By choosing $\epsilon$ such that $\epsilon=\min _{i}\left\{\epsilon_{i}\right\}$, for $i \in\{1,2,3,4,5\}$, we get

$$
S(t), v(0, t), e(0, t), i(0, t), R \geq \epsilon \text {. }
$$

\section{Local Stability of Equilibria}

The conditions of stability for each equilibrium will be derived through linearization technique around the equilibrium.

The conditions of stability for the disease-free equilibrium $E^{0}$ can be investigated through the following result.

Theorem 12. If $\Re_{0}<1$ then $E^{0}$ is locally asymptotically stable; if $\mathfrak{R}_{0}>1$ then $E^{0}$ is unstable.

Proof. To investigate the stability of the disease-free equilibrium $E^{0}$, we denote by $\widetilde{S}(t), \widetilde{v}(a, t), \widetilde{e}(a, t), \widetilde{i}(a, t)$, and $\widetilde{R}(t)$ the perturbations of $S(t), v(a, t), e(a, t), i(a, t)$, and $R(t)$, respectively, such that

$$
\begin{aligned}
\widetilde{S}(t) & =S(t)-S^{0}, \\
\widetilde{v}(a, t) & =v(a, t)-v^{0}(a) \\
\widetilde{e}(a, t) & =e(a, t), \\
\widetilde{i}(a, t) & =i(a, t), \\
\widetilde{R}(t) & =R(t) .
\end{aligned}
$$

The perturbations satisfy the following:

$$
\begin{aligned}
& \frac{\mathrm{d}}{\mathrm{d} t} \widetilde{S}(t)=-\left(\nu+\mu^{0}\right) \widetilde{S}(t)+\int_{0}^{\infty} \alpha(a) \widetilde{v}(a, t) d a \\
& \quad-S^{0} \int_{0}^{\infty}\left(K_{0}(a) \widetilde{i}(a, t)\right. \\
& \left.\quad+\int_{0}^{\infty} K\left(a, a^{\prime}\right) \widetilde{i}\left(a^{\prime}, t\right) d a^{\prime}\right) d a \\
& \frac{\partial}{\partial t} \widetilde{v}(a, t)=-\frac{\partial}{\partial a} \widetilde{v}(a, t)-\eta(a) \widetilde{v}(a, t) \\
& \frac{\partial}{\partial t} \widetilde{e}(a, t)=-\frac{\partial}{\partial a} \widetilde{e}(a, t)-\varrho(a) \widetilde{e}(a, t) \\
& \frac{\partial}{\partial t} \widetilde{i}(a, t)=-\frac{\partial}{\partial a} \tilde{i}(a, t)-\sigma(a) \widetilde{i}(a, t) \\
& \frac{\mathrm{d}}{\mathrm{d} t} \widetilde{R}=\int_{0}^{\infty} \gamma(a) \widetilde{i}(a, t) d a-\mu^{0} \widetilde{R},
\end{aligned}
$$

after substituting (81) into (4) and neglecting the terms of order higher or equal to two, with boundary conditions

$$
\begin{aligned}
& \widetilde{v}(0, t)=v \widetilde{S}(t) \\
& \widetilde{e}(0, t)=S^{0} \int_{0}^{\infty}\left(K_{0}(a) \tilde{i}(a, t)\right. \\
& \left.+\int_{0}^{\infty} K\left(a, a^{\prime}\right) \widetilde{i}\left(a^{\prime}, t\right) d a^{\prime}\right) d a \\
& \widetilde{i}(0, t)=\int_{0}^{\infty} \varepsilon(a) \widetilde{e}(a, t) d a,
\end{aligned}
$$

after substituting (81) into (5) and neglecting the terms of order higher or equal to two.

Now, we consider the exponential solutions of systems (82)-(83) of the form

$$
\begin{aligned}
\widetilde{S}(t) & =\bar{S} e^{\lambda t}, \\
\widetilde{v}(a, t) & =\bar{v}(a) e^{\lambda t}, \\
\widetilde{e}(a, t) & =\bar{e}(a) e^{\lambda t}, \\
\widetilde{i}(a, t) & =\bar{i}(a) e^{\lambda t}, \\
\widetilde{R}(t) & =\bar{R} e^{\lambda t},
\end{aligned}
$$


where $\bar{S}, \bar{v}(a), \bar{e}(a), \bar{i}(a)$, and $\lambda$ (real or complex number) satisfy the following system:

$$
\begin{aligned}
& \lambda \bar{S}=-\left(\nu+\mu^{0}\right) \bar{S}+\int_{0}^{\infty} \alpha(a) \bar{v}(a) d a \\
& -S^{0} \int_{0}^{\infty}\left(K_{0}(a) \bar{i}(a)\right. \\
& \left.\quad+\int_{0}^{\infty} K\left(a, a^{\prime}\right) \bar{i}\left(a^{\prime}\right) d a^{\prime}\right) d a \\
& \lambda \bar{v}(a)=-\frac{d}{d a} \bar{v}(a)-\eta(a) \bar{v}(a) \\
& \lambda \bar{e}(a)=-\frac{d}{d a} \bar{e}(a)-\varrho(a) \bar{e}(a) \\
& \lambda \bar{i}(a)=-\frac{d}{d a} \bar{i}(a)-\sigma(a) \bar{i}(a) \\
& \lambda \bar{R}=\int_{0}^{\infty} \gamma(a) \bar{i}(a) d a-\mu^{0} \bar{R},
\end{aligned}
$$

with boundary conditions

$$
\bar{v}(0)=v \bar{S}
$$

$$
\begin{aligned}
& =S^{0} \int_{0}^{\infty}\left(K_{0}(a) \bar{i}(a)+\int_{0}^{\infty} K\left(a, a^{\prime}\right) \bar{i}\left(a^{\prime}\right) d a^{\prime}\right) d a \\
& \bar{i}(0)=\int_{0}^{\infty} \varepsilon(a) \bar{e}(a) d a .
\end{aligned}
$$

From the second, third, and fourth equations of (85), we get

$$
\begin{aligned}
& \bar{v}(a)=\bar{v}(0) e^{-\lambda a-\int_{0}^{a} \eta(s) d s}, \\
& \bar{e}(a)=\bar{e}(0) e^{-\lambda a-\int_{0}^{a} \varrho(s) d s}, \\
& \bar{i}(a)=\bar{i}(0) e^{-\lambda a-\int_{0}^{a} \sigma(s) d s},
\end{aligned}
$$

respectively, where $\bar{v}(0), \bar{e}(0)$, and $\bar{i}(0)$ are given by (86).

Substituting the last equation of (87) into the boundary condition given by the second equation of (86) yields the characteristic equation

$$
\mathfrak{C}^{0}(\lambda)=1,
$$

where

$$
\begin{aligned}
& \mathfrak{S}^{0}(\lambda)=S^{0}\left(\int_{0}^{\infty} \mathcal{E}(a) e^{-\lambda a-\int_{0}^{a} \varrho(s) d s} d a\right) \\
& \times \int_{0}^{\infty}\left(K_{0}(a) e^{-\lambda a-\int_{0}^{a} \sigma(s) d s}\right. \\
& \left.+\int_{0}^{\infty} K\left(a, a^{\prime}\right) e^{-\lambda a^{\prime}-\int_{0}^{a^{\prime}} \sigma(s) d s} d a^{\prime}\right) d a,
\end{aligned}
$$

such that $\mathfrak{C}^{0}(0)=\mathfrak{R}_{0}$. It is not difficult to see that $(d / d \lambda) \mathfrak{C}^{0}(\lambda)=-\mathfrak{V}^{0}(\lambda)<0$. Thus, $\mathfrak{F}^{0}(\lambda)$ is a decreasing continuous function of $\lambda$ which approaches $\infty$ as $\lambda \longrightarrow-\infty$ and 0 as $\lambda \longrightarrow \infty$. Hence, the characteristic equation (88) admits a real solution $\lambda^{*}$ such that $\lambda^{*}<0$ whenever $\mathfrak{S}^{0}(0)<$ 1 and $\lambda^{*}>0$ whenever $\mathfrak{S}^{0}(0)>1$.

On the other hand, by assuming a complex solution $\lambda=$ $\alpha+i \beta$ of the characteristic equation $\mathfrak{C}^{0}(\lambda)=1$, we can notice that $\Re\left(e^{\lambda}\right) \leq e^{\Re(\lambda)}$ is always true. Thus, we clearly get $\mathfrak{R} \mathfrak{C}^{0}(\lambda) \leq \mathfrak{C}^{0}(\mathfrak{R} \lambda)$. It follows from the characteristic equation $\mathfrak{C}^{0}(\lambda)=1$ that $\mathfrak{R} \mathfrak{C}^{0}(\lambda)=1$ and $\mathfrak{I} \mathfrak{C}^{0}(\lambda)=0$. Therefore, we obtain $1 \leq \mathfrak{C}^{0}(\mathfrak{R} \lambda)$, i.e., $\mathfrak{C}^{0}\left(\lambda^{*}\right) \leq \mathfrak{C}^{0}(\mathfrak{R} \lambda)$. Hence, $\mathfrak{R} \lambda \leq \lambda^{*}$, since $\mathfrak{C}^{0}(\lambda)$ is a decreasing function.

It results from the above statements that all eigenvalues of the characteristic equation $\mathfrak{S}^{0}(\lambda)=1$ have negative real part whenever $\mathfrak{C}^{0}(0)<1$, i.e., $\mathfrak{R}_{0}<1$. Thus, the diseasefree equilibrium $E^{0}$ is locally asymptotically stable if $\mathfrak{R}_{0}<$ 1. Otherwise, $\mathfrak{c}^{0}(0) \geq 1$, i.e., the unique real solution of the characteristic equation $\mathfrak{C}^{0}(\lambda)=1$ is positive, and hence the disease-free equilibrium $E^{0}$ is unstable.

Theorem 13. If $\Re_{0}>1$ then $E^{*}$ is locally asymptotically stable.

Proof. Likewise for the disease-free equilibrium, we perturb the disease-endemic equilibrium by letting

$$
\begin{aligned}
\widetilde{S}(t) & =S(t)-S^{*}, \\
\widetilde{v}(a, t) & =v(a, t)-v^{*}(a), \\
\widetilde{e}(a, t) & =e(a, t)-e^{*}(a), \\
\widetilde{i}(a, t) & =i(a, t)-i^{*}(a), \\
\widetilde{R}(t) & =R(t)-R^{*} .
\end{aligned}
$$

Substituting $S(t)=\widetilde{S}(t)+S^{*}, v(a, t)=\widetilde{v}(a, t)+v^{*}(a), e(a, t)=$ $\widetilde{e}(a, t)+e^{*}(a)$, and $i(a, t)=\widetilde{i}(a, t)+i^{*}(a), R(t)=\widetilde{R}(t)+R^{*}$ into (4) and neglecting the terms of second order and above, the perturbations satisfy the linear system

$$
\begin{aligned}
& \frac{\mathrm{d}}{\mathrm{d} t} \widetilde{S}(t)=-\left(\nu+\mu^{0}\right) \widetilde{S}(t)+\int_{0}^{\infty} \alpha(a) \widetilde{v}(a, t) d a \\
& -S^{*} \int_{0}^{\infty}\left(K_{0}(a) \widetilde{i}(a, t)\right. \\
& \left.\quad+\int_{0}^{\infty} K\left(a, a^{\prime}\right) \widetilde{i}\left(a^{\prime}, t\right) d a^{\prime}\right) d a-\widetilde{S}(t) \\
& \quad \cdot \int_{0}^{\infty}\left(K_{0}(a) i^{*}(a)\right. \\
& \left.\quad+\int_{0}^{\infty} K\left(a, a^{\prime}\right) i^{*}\left(a^{\prime}\right) d a^{\prime}\right) d a
\end{aligned}
$$




$$
\begin{aligned}
& \frac{\partial}{\partial t} \widetilde{v}(a, t)=-\frac{\partial}{\partial a} \widetilde{v}(a, t)-\eta(a) \widetilde{v}(a, t) \\
& \frac{\partial}{\partial t} \widetilde{e}(a, t)=-\frac{\partial}{\partial a} \widetilde{e}(a, t)-\varrho(a) \widetilde{e}(a, t) \\
& \frac{\partial}{\partial t} \tilde{i}(a, t)=-\frac{\partial}{\partial a} \widetilde{i}(a, t)-\sigma(a) \widetilde{i}(a, t) \\
& \frac{\mathrm{d}}{\mathrm{d} t} \widetilde{R}=\int_{0}^{\infty} \gamma(a) \widetilde{i}(a, t) d a-\mu^{0} \widetilde{R},
\end{aligned}
$$

with boundary conditions

$$
\begin{aligned}
& \widetilde{v}(0, t)=\nu \widetilde{S}(t) \\
& \widetilde{e}(0, t)=S^{*} \int_{0}^{\infty}\left(K_{0}(a) \tilde{i}(a, t)\right. \\
& \left.+\int_{0}^{\infty} K\left(a, a^{\prime}\right) \widetilde{i}\left(a^{\prime}, t\right) d a^{\prime}\right) d a+\widetilde{S}(t) \\
& \cdot \int_{0}^{\infty}\left(K_{0}(a) i^{*}(a)\right. \\
& \left.+\int_{0}^{\infty} K\left(a, a^{\prime}\right) i^{*}\left(a^{\prime}\right) d a^{\prime}\right) d a \\
& \widetilde{i}(0, t)=\int_{0}^{\infty} \varepsilon(a) \widetilde{e}(a, t) d a,
\end{aligned}
$$

after substituting $S(t)=\widetilde{S}(t)+S^{*}, v(a, t)=\widetilde{v}(a, t)+v^{*}(a)$, $e(a, t)=\widetilde{e}(a, t)+e^{*}(a), i(a, t)=\widetilde{i}(a, t)+i^{*}(a)$, and $R(t)=$ $\widetilde{R}(t)+R^{*}$ into (5) and neglecting the terms of second order and above.

Now, we consider the exponential solutions of systems (91)-(92) of the form

$$
\begin{aligned}
\widetilde{S}(t) & =\bar{S} e^{\lambda t}, \\
\widetilde{v}(a, t) & =\bar{v}(a) e^{\lambda t}, \\
\widetilde{e}(a, t) & =\bar{e}(a) e^{\lambda t}, \\
\widetilde{i}(a, t) & =\bar{i}(a) e^{\lambda t}, \\
\widetilde{R}(t) & =\bar{R} e^{\lambda t},
\end{aligned}
$$

where $\bar{S}, \bar{v}(a), \bar{e}(a), \bar{i}(a)$, and $\lambda$ (real or complex number) satisfy the system

$$
\begin{aligned}
& \lambda \bar{S}=-\left(\nu+\mu^{0}\right) \bar{S}+\int_{0}^{\infty} \alpha(a) \bar{v}(a) d a \\
& \quad-S^{*} \int_{0}^{\infty}\left(K_{0}(a) \bar{i}(a)\right. \\
& \left.+\int_{0}^{\infty} K\left(a, a^{\prime}\right) \bar{i}\left(a^{\prime}\right) d a^{\prime}\right) d a \\
& \quad-\bar{S} \int_{0}^{\infty}\left(K_{0}(a) i^{*}(a)\right. \\
& \left.\quad+\int_{0}^{\infty} K\left(a, a^{\prime}\right) i^{*}\left(a^{\prime}\right) d a^{\prime}\right) d a \\
& \lambda \bar{v}(a)=-\frac{\mathrm{d}}{\mathrm{d} a} \bar{v}(a)-\eta(a) \bar{v}(a) \\
& \lambda \bar{e}(a)=-\frac{\mathrm{d}}{\mathrm{d} a} \bar{e}(a)-\varrho(a) \bar{e}(a) \\
& \lambda \bar{i}(a)=-\frac{\mathrm{d}}{\mathrm{d} a} \bar{i}(a)-\sigma(a) \bar{i}(a) \\
& \lambda \bar{R}=\int_{0}^{\infty} \gamma(a) \bar{i}(a) d a-\mu^{0} \bar{R}
\end{aligned}
$$

with boundary conditions

$$
\begin{aligned}
& \bar{v}(0)=\nu \bar{S} \\
& \bar{e}(0)=S^{*} \int_{0}^{\infty}\left(K_{0}(a) \bar{i}(a)\right. \\
& \left.+\int_{0}^{\infty} K\left(a, a^{\prime}\right) \bar{i}\left(a^{\prime}\right) d a^{\prime}\right) d a \\
& +\bar{S} \int_{0}^{\infty}\left(K_{0}(a) i^{*}(a)\right. \\
& \left.+\int_{0}^{\infty} K\left(a, a^{\prime}\right) i^{*}\left(a^{\prime}\right) d a^{\prime}\right) d a \\
& \bar{i}(0)=\int_{0}^{\infty} \varepsilon(a) \bar{e}(a) d a .
\end{aligned}
$$

Similarly to the process leading to the characteristic equation (88), the characteristic equation at the disease-endemic equilibrium $E^{*}$ is given by

$$
\left(\lambda+\mu^{0}+\nu A_{\lambda}\right) \mathfrak{S}^{*}(\lambda)-B-\left(\lambda+\mu^{0}+\nu A_{\lambda}\right)=0,
$$


where

$$
\begin{aligned}
& \mathfrak{S}^{*}(\lambda)=\frac{\mathfrak{S}^{0}(\lambda)}{\mathfrak{R}_{0}}>0 \\
& A_{\lambda}=1-\int_{0}^{\infty} \alpha(a) e^{-\lambda a-\int_{0}^{a} \eta(s) d s} d a>0
\end{aligned}
$$

$B$

$$
\begin{aligned}
& =\int_{0}^{\infty}\left(K_{0}(a) i^{*}(a)+\int_{0}^{\infty} K\left(a, a^{\prime}\right) i^{*}\left(a^{\prime}\right) d a^{\prime}\right) d a \\
& >0 .
\end{aligned}
$$

It is sufficient to prove that (96) has no root with nonnegative real part. So, we suppose that (96) has a complex root with nonnegative real part denoted by

$$
\lambda=\alpha+i \beta
$$

where $\alpha \geq 0$ and $\beta \neq 0$. It follows from (96) that

$$
\begin{gathered}
\left(\alpha+i \beta+\mu^{0}+v A_{\alpha+i \beta}\right) \mathfrak{E}^{*}(\alpha+i \beta)-B \\
-\left(\alpha+i \beta+\mu^{0}+v A_{\alpha+i \beta}\right)=0
\end{gathered}
$$

where

$$
\begin{aligned}
& \mathfrak{S}^{*}(\alpha+i \beta)=\frac{\mathfrak{C}^{0}(\alpha+i \beta)}{\mathfrak{R}_{0}}>0 \\
& A_{\alpha+i \beta}=1-\int_{0}^{\infty} \alpha(a) e^{-\alpha a-\int_{0}^{a} \eta(s) d s} e^{-i \beta a} d a>0 \\
& B \\
& =\int_{0}^{\infty}\left(K_{0}(a) i^{*}(a)+\int_{0}^{\infty} K\left(a, a^{\prime}\right) i^{*}\left(a^{\prime}\right) d a^{\prime}\right) d a \\
& >0,
\end{aligned}
$$

and hence

$$
\mathfrak{C}^{*}(\alpha+i \beta)=\mathfrak{R} \mathfrak{C}^{*}(\alpha+i \beta)+\mathfrak{I} \mathfrak{S}^{*}(\alpha+i \beta),
$$

where

$$
\begin{aligned}
& \mathfrak{R} \boldsymbol{C}^{*}(\alpha+i \beta)=1+\frac{B}{K_{\alpha \beta}}\left(\alpha+\mu^{0}+v\right. \\
& \left.-v \int_{0}^{\infty} \alpha(a) e^{-\alpha a-\int_{0}^{a} \eta(s) d s} \cos \beta a d a\right)>1
\end{aligned}
$$

and

$$
\begin{aligned}
& \mathfrak{J C}^{*}(\alpha+i \beta) \\
& \quad=-\frac{B}{K_{\alpha \beta}}\left(\beta-v \int_{0}^{\infty} \alpha(a) e^{-\alpha a-\int_{0}^{a} \eta(s) d s} \sin \beta a d a\right) \\
& \quad<0
\end{aligned}
$$

with

$$
\begin{aligned}
K_{\alpha \beta} & =\left(\alpha+\mu^{0}+\nu\right. \\
& \left.-\nu \int_{0}^{\infty} \alpha(a) e^{-\alpha a-\int_{0}^{a} \eta(s) d s} \cos \beta a d a\right)^{2}+(\beta \\
& \left.-v \int_{0}^{\infty} \alpha(a) e^{-\alpha a-\int_{0}^{a} \eta(s) d s} \sin \beta a d a\right)^{2}>0 .
\end{aligned}
$$

Since $\mathfrak{R} \mathfrak{C}^{*}(\alpha+i \beta) \leq \mathfrak{C}^{*}(\alpha)$ is always true and $\mathfrak{C}^{*}(\alpha) \leq$ $\mathfrak{C}^{*}(0)=1\left(\mathfrak{C}^{*}\right.$ is a decreasing function of $\left.\lambda\right)$, thus $\mathfrak{R} \mathfrak{S}^{*}(\alpha+$ iß) $\leq 1$.

Therefore, the latter statement contradicts (102).

\section{Global Stability of Equilibria}

To investigate the global asymptotic stability of equilibria of system (4) we shall use suitable Volterra-type Lyapunov functions of the form

$$
G(X)=X-1-\ln X, \quad X>0 .
$$

We state the following results.

Theorem 14. The disease-free equilibrium $E^{0}$ of (4) is globally asymptotically stable if $\mathfrak{R}_{0} \leq 1$.

Proof. A Lyapunov function $L^{0}$ of the form

$$
L^{0}(t)=L_{1}^{0}(t)+L_{2}^{0}(t)+L_{3}^{0}(t)+L_{4}^{0}(t)+L_{5}^{0}(t)
$$

is considered, where

$$
\begin{aligned}
& L_{1}^{0}(t)=S^{0} G\left(\frac{S(t)}{S^{0}}\right), \\
& L_{2}^{0}(t)=\int_{0}^{\infty} v^{0}(a) G\left(\frac{v(a, t)}{v^{0}(a)}\right) d a \\
& L_{3}^{0}(t)=\int_{0}^{\infty} \varphi(a) e(a, t) d a, \\
& L_{4}^{0}(t)=\int_{0}^{\infty} \omega(a) i(a, t) d a, \\
& L_{5}^{0}(t)=C^{0} R(t) .
\end{aligned}
$$

The functions $\varphi$ and $\omega$ are nonnegative functions to be chosen suitably and carefully. $\dot{L}_{i}^{0}, i=1, \ldots, 5$, denote the derivatives 
Abstract and Applied Analysis

15

of $L_{i}^{0}$ with respect to $t$ along the solution to (4) and are given by

$$
\begin{aligned}
& \dot{L}_{1}^{0}(\cdot)=\left(\nu+\mu^{0}\right) S^{0}\left(2-\frac{S^{0}}{S}-\frac{S}{S^{0}}\right)+\left(1-\frac{S^{0}}{S}\right) \\
& \cdot \int_{0}^{\infty} \alpha(a) v(a, \cdot)\left(1-\frac{v^{0}(a)}{v(a, \cdot)}\right) d a \\
& +S^{0} \int_{0}^{\infty}\left(K_{0}(a) i(a, \cdot)\right. \\
& \left.+\int_{0}^{\infty} K\left(a, a^{\prime}\right) i\left(a^{\prime}, \cdot\right) d a^{\prime}\right) d a-e(0, \cdot) \\
& \dot{L}_{2}^{0}(\cdot)=-\lim _{a \longrightarrow \infty} v(a, \cdot)+v\left(S-S^{0} \ln S\right)+v S^{0}(1 \\
& -\ln v)-\int_{0}^{\infty} \eta(a)\left(v(a, \cdot)-v^{0}(a) \ln v(a, \cdot)\right) d a, \\
& \dot{L}_{3}^{0}(\cdot)=-\lim _{a \longrightarrow \infty} \varphi(a) e(a, \cdot)+\varphi(0) e(0, \cdot) \\
& +\int_{0}^{\infty}(\dot{\varphi}(a)-\varrho(a) \varphi(a)) e(a, \cdot) d a, \\
& \dot{L}_{4}^{0}(\cdot)=-\lim _{a \longrightarrow \infty} \omega(a) i(a, \cdot)+\omega(0) i(0, \cdot) \\
& +\int_{0}^{\infty}(\dot{\omega}(a)-\sigma(a) \omega(a)) i(a, \cdot) d a, \\
& \dot{L}_{5}^{0}(\cdot)=C^{0} \int_{0}^{\infty} \gamma(a) i(a, \cdot) d a-C^{0} \mu^{0} R .
\end{aligned}
$$

Therefore,

$$
\begin{gathered}
\dot{L}^{0}(\cdot)=\left(\nu+\mu^{0}\right) S^{0}\left(2-\frac{S^{0}}{S}-\frac{S}{S^{0}}\right)+(\varphi(0)-1) \\
\cdot e(0, \cdot)+\nu\left(S-S^{0} \ln S\right)+\nu S^{0}(1-\ln v)-(1 \\
\left.-\frac{S^{0}}{S}\right) \int_{0}^{\infty} \alpha(a) v^{0}(a) d a-C^{0}\left(\mu^{0} R\right. \\
\left.-\int_{0}^{\infty} \gamma(a) i(a, \cdot) d a\right) \\
-\frac{S^{0}}{S} \int_{0}^{\infty} \alpha(a) v(a, \cdot) d a+\int_{0}^{\infty} \alpha(a) v(a, \cdot) d a \\
-\int_{0}^{\infty} \eta(a)\left(v(a, \cdot)-v^{0}(a) \ln v(a, \cdot)\right) d a
\end{gathered}
$$

$$
\begin{aligned}
& +\int_{0}^{\infty}\left(\omega^{\prime}(a)-\sigma(a) \omega(a)+S^{0} K_{0}(a)\right. \\
& \left.+S^{0} \int_{0}^{\infty} K\left(a, a^{\prime}\right) \frac{i\left(a^{\prime}, \cdot\right)}{i(a, \cdot)}\right) i(a, \cdot) d a \\
& +\int_{0}^{\infty}\left(\varphi^{\prime}(a)-\varrho(a) \varphi(a)+\omega(0) \varepsilon(a)\right) \\
& \cdot e(a, \cdot) d a,
\end{aligned}
$$

i.e.,

$$
\begin{aligned}
& \dot{L}^{0}(\cdot)=\left(\nu+\mu^{0}\right) S^{0}\left(2-\frac{S^{0}}{S}-\frac{S}{S^{0}}\right)+(\varphi(0)-1) \\
& \cdot e(0, \cdot)+v\left(S-S^{0} \ln S\right)+\nu S^{0}(1-\ln v)-(1 \\
& \left.-\frac{S^{0}}{S}\right) \int_{0}^{\infty} \alpha(a) v^{0}(a) d a-C^{0}\left(\mu^{0} R\right. \\
& \left.-\int_{0}^{\infty} \gamma(a) i(a, \cdot) d a\right) \\
& -\frac{S^{0}}{S} \int_{0}^{\infty} \alpha(a) v(a, \cdot) d a+\int_{0}^{\infty} \alpha(a) v(a, \cdot) d a \\
& -\int_{0}^{\infty} \eta(a)\left(v(a, \cdot)-v^{0}(a) \ln v(a, \cdot)\right) d a \\
& +\int_{0}^{\infty}\left(\omega^{\prime}(a)-\sigma(a) \omega(a)+S^{0} K_{0}(a)\right. \\
& \left.+S^{0} \int_{0}^{\infty} K\left(a, a^{\prime}\right) e^{-\int_{a}^{a^{\prime}} \sigma(s) d s} d a^{\prime}\right) i(a, \cdot) d a \\
& +\int_{0}^{\infty}\left(\varphi^{\prime}(a)-\varrho(a) \varphi(a)+\omega(0) \varepsilon(a)\right) \\
& \cdot e(a, \cdot) d a,
\end{aligned}
$$

after choosing $a$ and $a^{\prime}$ such that

$$
\frac{i\left(a^{\prime}, t\right)}{\zeta\left(a^{\prime}\right)}=\frac{i(a, t)}{\zeta(a)} .
$$

Note that

$$
\begin{aligned}
\nu(S & \left.-S^{0} \ln S\right)+v S^{0}(1-\ln v)-\left(1-\frac{S^{0}}{S}\right) \\
\cdot & \int_{0}^{\infty} \alpha(a) v^{0}(a) d a=-v S^{0}(1+\ln \nu S) \\
+ & \frac{1}{S}\left[\nu S^{2}+\left(2 \nu S^{0}+\left(\Lambda-\left(\nu+\mu^{0}\right) S^{0}\right)\right) S\right. \\
& \left.-\left(\Lambda-\left(\nu+\mu^{0}\right) S^{0}\right) S^{0}\right],
\end{aligned}
$$


after using $\int_{0}^{\infty} \alpha(a) v^{0}(a) d a=-\Lambda+\left(\nu+\mu^{0}\right) S^{0}$. Thus, (110) becomes

$$
\begin{aligned}
& \dot{L}^{0}(\cdot)=\left(\nu+\mu^{0}\right) S^{0}\left(2-\frac{S^{0}}{S}-\frac{S}{S^{0}}\right)+(\varphi(0)-1) \\
& \cdot e(0, \cdot)-C^{0}\left(\mu^{0} R-\int_{0}^{\infty} \gamma(a) i(a, \cdot) d a\right)-\nu S^{0}(1 \\
& +\ln \nu S)+\frac{1}{S}\left[\nu S^{2}+\left(2 \nu S^{0}+\left(\Lambda-\left(\nu+\mu^{0}\right) S^{0}\right)\right) S\right. \\
& \left.-\left(\Lambda-\left(\nu+\mu^{0}\right) S^{0}\right) S^{0}\right] \\
& -\frac{S^{0}}{S} \int_{0}^{\infty} \alpha(a) v(a, \cdot) d a-\int_{0}^{\infty}((\eta(a)-\alpha(a)) v(a, \cdot) \\
& \left.-\eta(a) v^{0}(a) \ln v(a, \cdot)\right) d a+\int_{0}^{\infty}\left(\omega^{\prime}(a)\right. \\
& \quad-\sigma(a) \omega(a)+S^{0} K_{0}(a) \\
& \left.+S^{0} \int_{0}^{\infty} K\left(a, a^{\prime}\right) e^{-\int_{a}^{a^{\prime}} \sigma(s) d s} d a^{\prime}\right) i(a, \cdot) d a \\
& +\int_{0}^{\infty}\left(\varphi^{\prime}(a)-\varrho(a) \varphi(a)+\omega(0) \varepsilon(a)\right) \\
& \cdot e(a, \cdot) d a .
\end{aligned}
$$

Using assumption A2, we show that

$$
\begin{aligned}
& \mu^{0} R(t)-\int_{0}^{\infty} \gamma(a) i(a, t) d a \\
& \quad \geq \mu^{0} R(t)-\bar{\gamma} \int_{0}^{\infty} i(a, t) d a \geq \mu^{0} R(t)-\gamma^{0} I(t) \\
& \quad>0,
\end{aligned}
$$

where

$$
I(t)=\int_{0}^{\infty} i(a, \cdot) d a .
$$

By assumption A3, obtain

$$
\begin{aligned}
& \int_{0}^{\infty}\left((\eta(a)-\alpha(a)) v(a, \cdot)-\eta(a) v^{0}(a) \ln v(a, \cdot)\right) d a \\
& \quad>\int_{0}^{\infty}\left(\eta(a)\left(1-v^{0}(a)\right)-\alpha(a)\right) v(a, \cdot) d a \\
& \quad>\int_{0}^{\infty}\left(\eta(a)\left(1-v S^{0}\right)-\alpha(a)\right) v(a, \cdot) d a \\
& \quad>\int_{0}^{\infty}(\eta(a) \\
& \cdot\left(1-\Lambda\left(1-\int_{0}^{\infty} \alpha(a) e^{-\int_{0}^{a} \eta(s) d s} d a\right)^{-1}\right) \\
& -\alpha(a)) v(a, \cdot) d a>0 .
\end{aligned}
$$

Next, we choose functions $\omega$ and $\varphi$ such that

$$
\begin{aligned}
& \omega(a) \\
& =S^{0} \int_{0}^{\infty}\left(K_{0}(u)+\int_{0}^{\infty} K\left(u, a^{\prime}\right) e^{-\int_{u}^{a^{\prime}} \sigma(s) d s} d a^{\prime}\right) \\
& \cdot e^{-\int_{a}^{u} \sigma(s) d s} d u
\end{aligned}
$$

and

$$
\varphi(a)=\omega(0) \int_{0}^{\infty} \varepsilon(u) e^{-\int_{a}^{u} \varrho(s) d s} d u .
$$

It follows from (117) and (118) that $\varphi(0)=\mathfrak{R}_{0}$ and

$$
\begin{aligned}
& \omega(0)=S^{0} \int_{0}^{\infty}\left(K_{0}(u) e^{-\int_{0}^{u} \sigma(s) d s}\right. \\
& \left.+\int_{0}^{\infty} K\left(u, a^{\prime}\right) e^{-\int_{0}^{a^{\prime}} \sigma(s) d s} d a^{\prime}\right) d u .
\end{aligned}
$$

Moreover, by differentiation of (117) and (118) with respect to age $a$, we obtain

$$
\begin{aligned}
\omega^{\prime}(a) & -\sigma(a) \omega(a)+S^{0} K_{0}(a) \\
+ & S^{0} \int_{0}^{\infty} K\left(a, a^{\prime}\right) e^{-\int_{a}^{a^{\prime}} \sigma(s) d s} d a^{\prime}=0
\end{aligned}
$$

and

$$
\varphi^{\prime}(a)-\varrho(a) \varphi(a)+\omega(0) \varepsilon(a),
$$

respectively. Therefore, (113) is reduced to

$$
\begin{aligned}
& \dot{L}^{0}(\cdot)=-\frac{\left(\nu+\mu^{0}\right)}{S}\left(S-S^{0}\right)^{2}+\left(\mathfrak{R}_{0}-1\right) e(0, \cdot) \\
& -C^{0}\left(\mu^{0} R-\int_{0}^{\infty} \gamma(a) i(a, \cdot) d a\right)-\nu S^{0}(1 \\
& \quad+\ln \nu S)+\frac{1}{S}\left[\nu S^{2}+\left(2 v S^{0}+\left(\Lambda-\left(\nu+\mu^{0}\right) S^{0}\right)\right) S\right. \\
& \left.\quad-\left(\Lambda-\left(\nu+\mu^{0}\right) S^{0}\right) S^{0}\right] \\
& -\frac{S^{0}}{S} \int_{0}^{\infty} \alpha(a) v(a, \cdot) d a-\int_{0}^{\infty}((\eta(a)-\alpha(a)) v(a, \cdot) \\
& \left.\quad-\eta(a) v^{0}(a) \ln v(a, \cdot)\right) d a .
\end{aligned}
$$

We denote

$$
\begin{aligned}
F(S)= & \nu S^{2}+\left(2 \nu S^{0}+\left(\Lambda-\left(\nu+\mu^{0}\right) S^{0}\right)\right) S \\
& -\left(\Lambda-\left(\nu+\mu^{0}\right) S^{0}\right) S^{0}
\end{aligned}
$$

Since

$$
\frac{\Lambda}{\mu^{0}+v}<S^{0}<\frac{\Lambda}{\mu^{0}-v}
$$


it is easy to check that $F(S)$ has two negative (real) roots. Moreover, $F(S)>0$ for every $S \geq 0$. Therefore, the sign of $\dot{L}^{0}(\cdot)$ will be determined by the sign of

$$
\begin{aligned}
& F(0)-\left(\nu+\mu^{0}\right)\left(S(t)-S^{0}\right)^{2} \\
& \quad=-\Lambda S^{0}-\left(\nu+\mu^{0}\right) S(t)\left(S(t)-2 S^{0}\right) .
\end{aligned}
$$

Thus, three cases occur:

Case $1\left(S(t)>2 S^{0}\right)$. It is easy to see that $F(0)-\left(\nu+\mu^{0}\right)(S(t)-$ $\left.S^{0}\right)^{2}<0$ and therefore $\dot{L}^{0}(t) \leq 0$ if $\Re_{0} \leq 1$.

Case $2\left(S(t)=2 S^{0}\right)$. For this value of $S(t)$, we have $F(0)-(\nu+$ $\left.\mu^{0}\right)\left(S(t)-S^{0}\right)^{2}=-\Lambda S^{0}<0$ and therefore $\dot{L}^{0}(t) \leq 0$ if $\mathfrak{R}_{0} \leq 1$.

Case $3\left(0<S(t)<2 S^{0}\right)$. For such values of $S(t)$ we have $F(0)-$ $\left(\nu+\mu^{0}\right)\left(S(t)-S^{0}\right)^{2}=-\left(\Lambda-2\left(\nu+\mu^{0}\right) S(t)\right) S^{0}-\left(\nu+\mu^{0}\right) S^{2}$. Since $2^{-1}\left(\nu+\mu^{0}\right)^{-1} \Lambda<2 S^{0}$, it follows that $F(0)-\left(\nu+\mu^{0}\right)\left(S(t)-S^{0}\right)^{2}<$ 0 for any $0<S(t)<2^{-1}\left(\nu+\mu^{0}\right)^{-1} \Lambda$. Therefore $\dot{L}^{0}(t) \leq 0$ if $\mathfrak{R}_{0} \leq 1$.

It results from the above that the derivative of $L^{0}(t)$ along the solutions of $(4)$ is $\dot{L}^{0}(t) \leq 0$. If $S(t)=S^{0}, v(a, t)=v^{0}(a)$, and $e(a, t)=i(a, t)=R(t)=0$ are simultaneously satisfied with $\mathfrak{R}_{0}=1$, then $\dot{L}^{0}(t)=0$ holds. Moreover, it can be verified that $\left\{(S, v, e, i, R): \dot{L}^{0}(t)=0\right\}=\left\{E^{0}\right\}$. Therefore, it results from Lasalle's Invariance Theorem [26, p. 200] that $E^{0}$ is globally asymptotically stable, if $\mathfrak{R}_{0} \leq 1$.

Theorem 15. The endemic equilibrium $E^{*}$ of (4) is globally asymptotically stable on the set, if $\Re_{0}>1$.

Proof. To prove the above result we consider a Lyapunov function of the form given below

$$
L^{*}(t)=L_{1}^{*}(t)+L_{2}^{*}(t)+L_{3}^{*}(t)+L_{4}^{*}(t)+L_{5}^{*}(t),
$$

where

$$
\begin{aligned}
& L_{1}^{*}(t)=S^{*} G\left(\frac{S(t)}{S^{*}}\right), \\
& L_{2}^{*}(t)=\int_{0}^{\infty} v^{*}(a) G\left(\frac{v(a, t)}{v^{*}(a)}\right) d a, \\
& L_{3}^{*}(t)=\int_{0}^{\infty} e^{*}(a) G\left(\frac{e(a, t)}{e^{*}(a)}\right) d a \\
& L_{4}^{*}(t)=\int_{0}^{\infty} i^{*}(a) G\left(\frac{i(a, t)}{i^{*}(a)}\right) d a, \\
& L_{5}^{*}(t)=R^{*} G\left(\frac{R(t)}{R^{*}}\right),
\end{aligned}
$$

with $G(X)=X-1-\ln X$, for any $X>0$.
Thus, we have

$$
\begin{aligned}
& \dot{L}_{1}^{*}(\cdot)=\left(1-\frac{S^{*}}{S}\right)\left[\Lambda-\left(\nu+\mu^{0}\right) S+\int_{0}^{\infty} \alpha(a)\right. \\
& \cdot v(a, \cdot) d a-S \int_{0}^{\infty}\left(K_{0}(a) i(a, \cdot)\right. \\
& \left.\left.\quad+\int_{0}^{\infty} K\left(a^{\prime}, a\right) i\left(a^{\prime}, \cdot\right) d a^{\prime}\right) d a\right]
\end{aligned}
$$

Using (37), (44), and (45) together with the first equation of (38), we obtain

$$
\begin{aligned}
& \dot{L}_{1}^{*}(\cdot)=\frac{\Lambda}{\mathfrak{R}_{0}}\left(2-\frac{S^{*}}{S}-\frac{S}{S^{*}}\right)+\left(2-\frac{S^{*}}{S}-\frac{S}{S^{*}}\right) \\
& \cdot \int_{0}^{\infty} \alpha(a) v^{*}(a) d a+\left(1-\frac{S^{*}}{S}\right)\left[-\int_{0}^{\infty} \alpha(a)\right. \\
& \cdot v^{*}(a) d a+\int_{0}^{\infty} \alpha(a) v(a, \cdot) d a \\
& -S \int_{0}^{\infty}\left(K_{0}(a) i(a, \cdot)\right. \\
& \left.+\int_{0}^{\infty} K\left(a^{\prime}, a\right) i\left(a^{\prime}, \cdot\right) d a^{\prime}\right) d a \\
& \quad-S^{*} \int_{0}^{\infty}\left(K_{0}(a) i^{*}(a)\right. \\
& \left.\left.+\int_{0}^{\infty} K\left(a^{\prime}, a\right) i^{*}\left(a^{\prime}\right) d a^{\prime}\right) d a\right] .
\end{aligned}
$$

We add and subtract, carefully, some terms to the above expression, and we also identify group of terms in the form given by $G(X)=X-1-\ln X$. We obtain

$$
\begin{aligned}
& \dot{L}_{1}^{*}(\cdot)=\frac{\Lambda}{\mathfrak{R}_{0}}\left(2-\frac{S^{*}}{S}-\frac{S}{S^{*}}\right)-\int_{0}^{\infty} \alpha(a) v^{*}(a) \\
& \cdot {\left[G\left(\frac{S^{*} v(a, \cdot)}{S v^{*}(a)}\right)-G\left(\frac{S^{*}}{S}\right)-G\left(\frac{v(a, \cdot)}{v^{*}(a)}\right)\right] d a } \\
&- 2 G\left(\frac{S^{*}}{S}\right) \int_{0}^{\infty} \alpha(a) v^{*}(a) d a-S^{*} \int_{0}^{\infty} K_{0}(a) \\
& \cdot i^{*}(a)\left[G\left(\frac{S i(a, \cdot)}{S^{*} i^{*}(a)}\right)-G\left(\frac{i(a, \cdot)}{i^{*}(a)}\right)\right] d a \\
&-S^{*} \int_{0}^{\infty} \int_{0}^{\infty} K\left(a^{\prime}, a\right) i^{*}\left(a^{\prime}\right) \\
& \cdot\left[G\left(\frac{S i\left(a^{\prime}, \cdot\right)}{S^{*} i^{*}\left(a^{\prime}\right)}\right)-G\left(\frac{i\left(a^{\prime}, \cdot\right)}{i^{*}\left(a^{\prime}\right)}\right)\right] d a^{\prime} d a .
\end{aligned}
$$

By differentiating $L_{2}^{*}$ with respect to $t$, we obtain

$$
\begin{gathered}
\dot{L}_{2}^{*}(\cdot)=-\int_{0}^{\infty}\left(1-\frac{v^{*}(a)}{v(a, \cdot)}\right) \\
\cdot\left(\frac{\partial}{\partial t} v(a, \cdot)+\eta(a) v(a, \cdot)\right) d a .
\end{gathered}
$$


Since

$$
\begin{aligned}
v^{*} & (a) \frac{\partial}{\partial t} G\left(\frac{v(a, \cdot)}{v^{*}(a)}\right) \\
\quad= & \left(1-\frac{v^{*}(a)}{v(a, \cdot)}\right)\left(\frac{\partial}{\partial t} v(a, \cdot)+\eta(a) v(a, \cdot)\right)
\end{aligned}
$$

thus (131) yields

$$
\dot{L}_{2}^{*}(\cdot)=-\int_{0}^{\infty} v^{*}(a) \frac{\partial}{\partial t} G\left(\frac{v(a, \cdot)}{v^{*}(a)}\right) d a .
$$

Using the second equation of (38) after integrating by part, we get

$$
\begin{aligned}
\dot{L}_{2}^{*}(\cdot)= & v^{*}(0) G\left(\frac{S}{S^{*}}\right) \\
& -\int_{0}^{\infty} \eta(a) v^{*}(a) G\left(\frac{v(a, \cdot)}{v^{*}(a)}\right) d a .
\end{aligned}
$$

Moreover,

$$
\begin{aligned}
v^{*} & (0) G\left(\frac{S}{S^{*}}\right) \\
& =v^{*}(0) \int_{0}^{\infty} \eta(a) e^{-\int_{0}^{\infty} \eta(s) d s} G\left(\frac{S}{S^{*}}\right) d a \\
& =\int_{0}^{\infty} \eta(a) v^{*}(a) G\left(\frac{S}{S^{*}}\right) d a,
\end{aligned}
$$

yields

$$
\begin{aligned}
& \dot{L}_{2}^{*}(\cdot) \\
& \quad=-\int_{0}^{\infty} \eta(a) v^{*}(a)\left[G\left(\frac{v(a, \cdot)}{v^{*}(a)}\right)-G\left(\frac{S}{S^{*}}\right)\right] d a .
\end{aligned}
$$

Similarly to $L_{2}^{*}$, from $L_{3}^{*}$ and $L_{4}^{*}$ we get, respectively,

$$
\begin{aligned}
& \dot{L}_{3}^{*}(\cdot)=-\int_{0}^{\infty} \varrho(a) e^{*}(a) \\
& \cdot\left[G\left(\frac{e(a, \cdot)}{e^{*}(a)}\right)-G\left(\frac{e(0, \cdot)}{e^{*}(0)}\right)\right] d a
\end{aligned}
$$

and

$$
\begin{aligned}
& \dot{L}_{4}^{*}(\cdot) \\
& =-\int_{0}^{\infty} \sigma(a) i^{*}(a)\left[G\left(\frac{i(a, \cdot)}{i^{*}(a)}\right)-G\left(\frac{i(0, \cdot)}{i^{*}(0)}\right)\right] d a,
\end{aligned}
$$

while using the fifth equation of $(38) L_{5}^{*}$ leads to

$$
\begin{aligned}
\dot{L}_{5}^{*}(\cdot) & =\mu^{0} R^{*}\left(2-\frac{R}{R^{*}}-\frac{R^{*}}{R}\right)-\int_{0}^{\infty} \gamma(a) i^{*}(a) \\
\cdot & {\left[G\left(\frac{R^{*} i(a, \cdot)}{R i^{*}(a)}\right)-G\left(\frac{R^{*}}{R}\right)\right.} \\
- & \left.G\left(\frac{i(a, \cdot)}{i^{*}(a)}\right)\right] d a .
\end{aligned}
$$

By combining (130), (136), (137), (138), and (139), we obtain

$$
\begin{aligned}
\dot{L}^{*}(\cdot) & =\frac{\Lambda}{\mathfrak{R}_{0}}\left(2-\frac{S^{*}}{S}-\frac{S}{S^{*}}\right)-\int_{0}^{\infty} \alpha(a) v^{*}(a) \\
\cdot & {\left[G\left(\frac{S^{*} v(a, \cdot)}{S v^{*}(a)}\right)-G\left(\frac{S^{*}}{S}\right)-G\left(\frac{v(a, \cdot)}{v^{*}(a)}\right)\right] d a } \\
& -2 G\left(\frac{S^{*}}{S}\right) \int_{0}^{\infty} \alpha(a) v^{*}(a) d a-S^{*} \int_{0}^{\infty} K_{0}(a) \\
\cdot & i^{*}(a)\left[G\left(\frac{S i(a, \cdot)}{S^{*} i^{*}(a)}\right)-G\left(\frac{i(a, \cdot)}{i^{*}(a)}\right)\right] d a \\
- & S^{*} \int_{0}^{\infty} \int^{\infty}\left(a^{\prime}, a\right) i^{*}\left(a^{\prime}\right) \\
& \cdot\left[G\left(\frac{S i\left(a^{\prime}, \cdot\right)}{S^{*} i^{*}\left(a^{\prime}\right)}\right)-G\left(\frac{i\left(a^{\prime}, \cdot\right)}{i^{*}\left(a^{\prime}\right)}\right)\right] d a^{\prime} d a \\
+ & \cdot \mu^{0} R^{*}\left(2-\frac{R}{R^{*}}-\frac{R^{*}}{R}\right)-\int_{0}^{\infty} \gamma(a) i i^{*}(a) \\
+ & G\left(\frac{S}{S^{*}}\right) \int_{0}^{\infty} \eta(a) v^{*}(a) d a-\int_{0}^{\infty} \eta(a) v^{*}(a) \\
& \cdot G\left(\frac{v(a, \cdot)}{v^{*}(a)}\right) d a-\int_{0}^{\infty} \varrho(a) e^{*}(a) \\
& \cdot\left[G\left(\frac{e(a, \cdot)}{e^{*}(a)}\right)-G\left(\frac{e(0, \cdot)}{e^{*}(0)}\right)\right] d a \\
& {\left.\left[\frac{i(a, \cdot)}{i^{*}(a)}\right)-G\left(\frac{i(0, \cdot)}{i^{*}(0)}\right)\right] d a }
\end{aligned}
$$

This yields

$$
\begin{aligned}
& \dot{L}^{*}(\cdot)=\frac{\Lambda}{R_{0}}\left(2-\frac{S^{*}}{S}-\frac{S}{S^{*}}\right)-\int_{0}^{\infty} \alpha(a) v^{*}(a) \\
& \cdot\left[G\left(\frac{S^{*} v(a, \cdot)}{S v^{*}(a)}\right)-G\left(\frac{S^{*}}{S}\right)\right. \\
& \left.-G\left(\frac{v(a, \cdot)}{v^{*}(a)}\right)\right] d a-S^{*} \int_{0}^{\infty} K_{0}(a) i^{*}(a) \\
& \cdot\left[G\left(\frac{S i(a, \cdot)}{S^{*} i^{*}(a)}\right)-G\left(\frac{S}{S^{*}}\right)-G\left(\frac{i(a, \cdot)}{i^{*}(a)}\right)\right] d a \\
& -S^{*} \int_{0}^{\infty} \int_{0}^{\infty} K\left(a^{\prime}, a\right) i^{*}\left(a^{\prime}\right)\left[G\left(\frac{S i\left(a^{\prime}, \cdot\right)}{S^{*} i^{*}\left(a^{\prime}\right)}\right)\right. \\
& \left.-G\left(\frac{S}{S^{*}}\right)-G\left(\frac{i\left(a^{\prime}, \cdot\right)}{i^{*}\left(a^{\prime}\right)}\right)\right] d a^{\prime} d a \\
& -2 G\left(\frac{S^{*}}{S}\right) \int_{0}^{\infty} \alpha(a) v^{*}(a) d a-\int_{0}^{\infty} \eta(a) v^{*}(a)
\end{aligned}
$$




$$
\begin{aligned}
& \cdot G\left(\frac{v(a, \cdot)}{v^{*}(a)}\right) d a-\left[S ^ { * } \int _ { 0 } ^ { \infty } \left(K_{0}(a) i^{*}(a)\right.\right. \\
& \left.+\int_{0}^{\infty} K\left(a^{\prime}, a\right) i^{*}\left(a^{\prime}\right) d a^{\prime}\right) d a-\int_{0}^{\infty} \eta(a) \\
& \left.\cdot v^{*}(a) d a\right] G\left(\frac{S}{S^{*}}\right)-\int_{0}^{\infty} \varrho(a) e^{*}(a) \\
& \cdot G\left(\frac{e^{*}(0) e(a, \cdot)}{e(0, t) e^{*}(a)}\right) d a-\int_{0}^{\infty} \varrho(a) e^{*}(a)(1 \\
& \left.-\frac{e^{*}(0)}{e(0, \cdot)}\right)\left(\frac{e(a, \cdot)}{e^{*}(a)}-\frac{e(0, \cdot)}{e^{*}(0)}\right) d a-\int_{0}^{\infty} \sigma(a) \\
& \cdot i^{*}(a) G\left(\frac{i^{*}(0) i(a, \cdot)}{i(0, t) i^{*}(a)}\right) d a-\int_{0}^{\infty} \sigma(a) i^{*}(a) \\
& \cdot\left(1-\frac{i^{*}(0)}{i(0, \cdot)}\right)\left(\frac{i(a, \cdot)}{i^{*}(a)}-\frac{i(0, \cdot)}{i^{*}(0)}\right) d a \\
& +\mu^{0} R^{*}\left(2-\frac{R}{R^{*}}-\frac{R^{*}}{R}\right)-\int_{0}^{\infty} \gamma(a) i^{*}(a) \\
& \cdot\left[G\left(\frac{R^{*} i(a, \cdot)}{R i^{*}(a)}\right)-G\left(\frac{R^{*}}{R}\right)\right. \\
& \left.-G\left(\frac{i(a, \cdot)}{i^{*}(a)}\right)\right] d a .
\end{aligned}
$$

Since

$$
\begin{aligned}
& S^{*} \int_{0}^{\infty}\left(K_{0}(a) i^{*}(a)+\int_{0}^{\infty} K\left(a^{\prime}, a\right) i^{*}\left(a^{\prime}\right) d a^{\prime}\right) d a \\
& \quad=1, \\
& \int_{0}^{\infty} \eta(a) e^{-\int_{0}^{\infty} \eta(s) d s} d a=1,
\end{aligned}
$$

thus

$$
\begin{aligned}
\dot{L}^{*}(\cdot) & =\frac{\Lambda}{\mathfrak{R}_{0}}\left(2-\frac{S^{*}}{S}-\frac{S}{S^{*}}\right)-\int_{0}^{\infty} \alpha(a) v^{*}(a) \\
\cdot & {\left[G\left(\frac{S^{*} v(a, \cdot)}{S v^{*}(a)}\right)-G\left(\frac{S^{*}}{S}\right)\right.} \\
- & \left.G\left(\frac{v(a, \cdot)}{v^{*}(a)}\right)\right] d a-S^{*} \int_{0}^{\infty} K_{0}(a) i^{*}(a) \\
\cdot & {\left[G\left(\frac{S i(a, \cdot)}{S^{*} i^{*}(a)}\right)-G\left(\frac{S}{S^{*}}\right)-G\left(\frac{i(a, \cdot)}{i^{*}(a)}\right)\right] d a } \\
& -S^{*} \int_{0}^{\infty} \int_{0}^{\infty} K\left(a^{\prime}, a\right) i^{*}\left(a^{\prime}\right)\left[G\left(\frac{S i\left(a^{\prime}, \cdot\right)}{S^{*} i^{*}\left(a^{\prime}\right)}\right)\right. \\
& \left.-G\left(\frac{S}{S^{*}}\right)-G\left(\frac{i\left(a^{\prime}, \cdot\right)}{i^{*}\left(a^{\prime}\right)}\right)\right] d a^{\prime} d a
\end{aligned}
$$

$$
\begin{aligned}
& -2 G\left(\frac{S^{*}}{S}\right) \int_{0}^{\infty} \alpha(a) v^{*}(a) d a-\int_{0}^{\infty} \eta(a) v^{*}(a) \\
& \cdot G\left(\frac{v(a, \cdot)}{v^{*}(a)}\right) d a-\left(1-\nu S^{*}\right) G\left(\frac{S}{S^{*}}\right) \\
& -\int_{0}^{\infty} \varrho(a) e^{*}(a) G\left(\frac{e^{*}(0) e(a, \cdot)}{e(0, t) e^{*}(a)}\right) d a \\
& -\int_{0}^{\infty} \varrho(a) e^{*}(a)\left(1-\frac{e^{*}(0)}{e(0, \cdot)}\right)\left(\frac{e(a, \cdot)}{e^{*}(a)}\right. \\
& \left.-\frac{e(0, \cdot)}{e^{*}(0)}\right) d a-\int_{0}^{\infty} \sigma(a) i^{*}(a) \\
& \cdot G\left(\frac{i^{*}(0) i(a, \cdot)}{i(0, t) i^{*}(a)}\right) d a-\int_{0}^{\infty} \sigma(a) i^{*}(a)(1 \\
& -\frac{i^{*}(0)}{i(0, \cdot)}\left(\frac{i(a, \cdot)}{i^{*}(a)}-\frac{i(0, \cdot)}{i^{*}(0)}\right) d a+\mu^{0} R^{*}(2 \\
& \left.-\frac{R}{R^{*}}-\frac{R^{*}}{R}\right)-\int_{0}^{\infty} \gamma(a) i^{*}(a)\left[G\left(\frac{R^{*} i(a, \cdot)}{R i^{*}(a)}\right)\right. \\
& \left.-G\left(\frac{R^{*}}{R}\right)-G\left(\frac{i(a, \cdot)}{i^{*}(a)}\right)\right] d a .
\end{aligned}
$$

Using assumption A3, it is easy to see that $\Lambda<1-$ $\int_{0}^{\infty} \alpha(a) e^{-\int_{0}^{a} \eta(s) d s} d a$. Hence, $S^{0} / \mathfrak{R}_{0}<1 / \nu$ for $\mathfrak{R}_{0}>1$; i.e., $1-\nu S^{*}>0$ for $\Re_{0}>1$. Moreover,

(i) $G\left(S^{*} v(a, \cdot) / S v^{*}(a)\right)-G\left(S^{*} / S\right)-G\left(v(a, \cdot) / v^{*}(a)\right)$ $=\left(1-S^{*} / S\right)\left(1-v(a, \cdot) / v^{*}(a)\right)>0 \Longleftrightarrow$ $\left(S / S^{*}<1\right.$ and $\left.v(a, \cdot) / v^{*}(a)>1\right)$ or $\left(S / S^{*}>\right.$ 1 and $\left.v(a, \cdot) / v^{*}(a)<1\right)$;

(ii) $G\left(S i(a, \cdot) / S^{*} i^{*}(a)\right)-G\left(S / S^{*}\right)-G\left(i(a, \cdot) / i^{*}(a)\right)$ $=\left(1-S / S^{*}\right)\left(1-i(a, \cdot) / i^{*}(a)\right)>0 \Longleftrightarrow$ $\left(S / S^{*}>1\right.$ and $\left.i(a, \cdot) / i^{*}(a)>1\right)$ or $\left(S / S^{*}<\right.$ 1 and $\left.i(a, \cdot) / i^{*}(a)<1\right)$;

(iii) $\left(1-e^{*}(a) / e(0, \cdot)\right)\left(e(a, \cdot) / e^{*}(a)-e(0, \cdot) / e^{*}(0)\right)>0 \Longleftrightarrow$ $\left(e(a, \cdot) / e^{*}(a)<e(0, \cdot) / e^{*}(0)<1\right)$ or $\left(e(a, \cdot) / e^{*}(a)>\right.$ $\left.e(0, \cdot) / e^{*}(0)>1\right)$;

(iv) $\left(1-i^{*}(a) / i(0, \cdot)\right)\left(i(a, \cdot) / i^{*}(a)-i(0, \cdot) / i^{*}(0)\right)>0 \Longleftrightarrow$ $\left(i(a, \cdot) / i^{*}(a)<i(0, \cdot) / i^{*}(0)<1\right)$ or $\left(i(a, \cdot) / i^{*}(a)>\right.$ $\left.i(0, \cdot) / i^{*}(0)>1\right)$;

(v) $G\left(R^{*} i(a, \cdot) / R i^{*}(a)\right)-G\left(R^{*} / R\right)-G\left(i(a, \cdot) / i^{*}(a)\right)$ $=\left(1-R^{*} / R\right)\left(1-i(a, \cdot) / i^{*}(a)\right)>0 \Longleftrightarrow$ $\left(R / R^{*}<1\right.$ and $\left.i(a, \cdot) / i^{*}(a)>1\right)$ or $\left(R / R^{*}>\right.$ 1 and $\left.i(a, \cdot) / i^{*}(a)<1\right)$.

Therefore, we obtain

$$
\dot{L}^{*}(t)<0,
$$

in the following cases:

(i) $S / S^{*}<1, v(a, \cdot) / v^{*}(a)>1, e(a, \cdot) / e^{*}(a)>$ $e(0, \cdot) / e^{*}(0)>1, i(a, \cdot) / i^{*}(a)<i(0, \cdot) / i^{*}(0)<1$, and $R / R^{*}>1$ 
(ii) $S / S^{*}<1, v(a, \cdot) / v^{*}(a)>1, e(a, \cdot) / e^{*}(a)<$ $e(0, \cdot) / e^{*}(0)<1, i(a, \cdot) / i^{*}(a)<i(0, \cdot) / i^{*}(0)<1$, and $R / R^{*}>1$

(iii) $S / S^{*}>1, v(a, \cdot) / v^{*}(a)<1, e(a, \cdot) / e^{*}(a)>$ $e(0, \cdot) / e^{*}(0)>1, i(a, \cdot) / i^{*}(a)>i(0, \cdot) / i^{*}(0)>1$, and $R / R^{*}<1$

(iv) $S / S^{*}>1, v(a, \cdot) / v^{*}(a)<1, e(a, \cdot) / e^{*}(a)<$ $e(0, \cdot) / e^{*}(0)<1, i(a, \cdot) / i^{*}(a)>i(0, \cdot) / i^{*}(0)>1$, and $R / R^{*}<1$.

From (144), we say that the derivative of $L^{*}(t)$ along the solutions of $(4)$ is $\dot{L}^{*}(t)<0$. If $S(t)=S^{*}, v(a, t)=$ $v^{*}(a), e(a, t)=e^{*}(a), i(a, t)=i^{*}(a)$, and $R(t)=R^{*}$ are simultaneously satisfied, then we obtain $\dot{L}^{*}(t)=0$ from (76). Moreover, it can be verified that $\left\{(S, v, e, i, R): \dot{L}^{*}(t)=0\right\}=$ $\left\{E^{*}\right\}$. Therefore, it results from Lasalle's Invariance Theorem $\left[26\right.$, p. 200] that $E^{*}$ is globally asymptotically stable, if $\boldsymbol{R}_{0}>$ 1.

The results from Theorems 14 and 15 show that there exists a threshold parameter, called the basic reproduction number and denoted by $\boldsymbol{R}_{0}$, that is essential in the stability analysis of the global dynamics of the model defined by system (4). Moreover, such a parameter can play a crucial role in the implementation of human vaccination policies. As we can see in

$$
\begin{aligned}
& \frac{\partial \Re_{0}}{\partial \nu}=-\frac{\Lambda(1-P)}{\left(\mu^{0}+\nu(1-P)\right)^{2}} \\
& \quad \cdot \int_{0}^{\infty}\left(K_{0}(a) \zeta(a)+\int_{0}^{\infty} K\left(a, a^{\prime}\right) \zeta\left(a^{\prime}\right) d a^{\prime}\right) d a \\
& \quad<0,
\end{aligned}
$$

the rise in rate of vaccination of (infant) susceptible individuals against an SEIR infection can reduce the spread of the infection and help to elaborate control measures to prevent and reduce the spread of the infection.

\section{Data Availability}

No data were used to support this study.

\section{Conflicts of Interest}

The authors declare that there are no conflicts of interest regarding the publication of this article.

\section{Acknowledgments}

The work done in this paper was funded by a research grant from Material Science Innovation and Modelling (MaSIM), North-West University, South Africa.

\section{References}

[1] J. Xu and Y. Zhou, "Global stability of a multi-group model with generalized nonlinear incidence and vaccination age," Discrete and Continuous Dynamical Systems - Series B, vol. 21, no. 3, pp. 977-996, 2016.

[2] R. Peralta, C. Vargas-De-León, and P. Miramontes, “Global Stability Results in a SVIR Epidemic Model with Immunity Loss Rate Depending on the Vaccine-Age," Abstract and Applied Analysis, vol. 2015, Article ID 341854, 8 pages, 2015.

[3] S. S. Chaves, P. Gargiullo, J. X. Zhang et al., "Loss of vaccineinduced immunity to varicella over time," The New England Journal of Medicine, vol. 356, no. 11, pp. 1121-1129, 2007.

[4] S. A. Plotkin, "Correlates of protection induced by vaccination," Clinical and Vaccine Immunology, vol. 17, no. 7, pp. 1055-1065, 2010.

[5] S. A. Plotkin, "Complex correlates of protection after vaccination," Clinical Infectious Diseases, vol. 56, no. 10, pp. 1458-1465, 2013.

[6] M. Prelog, "Differential approaches for vaccination from childhood to old age," Gerontology, vol. 59, no. 3, pp. 230-239, 2013.

[7] N. Wood and C.-A. Siegrist, "Neonatal immunization: where do we stand?" Current Opinion in Infectious Diseases, vol. 24, no. 3, pp. 190-195, 2011.

[8] S. M. Blower and A. R. McLean, "Prophylactic vaccines, risk behavior change, and the probability of eradicating HIV in San Francisco," Science, vol. 265, no. 5177, pp. 1451-1454, 1994.

[9] D. Ding and X. Ding, "Global stability of multi-group vaccination epidemic models with delays," Nonlinear Analysis: Real World Applications, vol. 12, no. 4, pp. 1991-1997, 2011.

[10] J. Q. Li, Y. L. Yang, and Y. C. Zhou, "Global stability of an epidemic model with latent stage and vaccination," Nonlinear Analysis: Real World Applications, vol. 12, no. 4, pp. 2163-2173, 2011.

[11] G. P. Sahu and J. Dhar, "Analysis of an SVEIS epidemic model with partial temporary immunity and saturation incidence rate," Applied Mathematical Modelling: Simulation and Computation for Engineering and Environmental Systems, vol. 36, no. 3, pp. 908-923, 2012.

[12] X. Song, Y. Jiang, and H. Wei, "Analysis of a saturation incidence SVEIRS epidemic model with pulse and two time delays," Applied Mathematics and Computation, vol. 214, no. 2, pp. 381390, 2009.

[13] J. Wang, J. Lang, and Y. Chen, "Global threshold dynamics of an SVIR model with age-dependent infection and relapse," Journal of Biological Dynamics, vol. 11, no. suppl. 2, pp. 427-454, 2017.

[14] J. Wang, R. Zhang, and T. Kuniya, "The stability analysis of an SVEIR model with continuous age-structure in the exposed and infectious classes," Journal of Biological Dynamics, vol. 9, no. 1, pp. 73-101, 2015.

[15] Y. Xiao and S. Tang, "Dynamics of infection with nonlinear incidence in a simple vaccination model," Nonlinear Analysis: Real World Applications, vol. 11, no. 5, pp. 4154-4163, 2010.

[16] Y. Yang, S. Tang, X. Ren, H. Zhao, and C. Guo, "Global stability and optimal control for a tuberculosis model with vaccination and treatment," Discrete and Continuous Dynamical Systems Series B, vol. 21, no. 3, pp. 1009-1022, 2016.

[17] X. Duan, S. Yuan, and X. Li, "Global stability of an SVIR model with age of vaccination," Applied Mathematics and Computation, vol. 226, pp. 528-540, 2014.

[18] M. Iannelli, M. Martcheva, and X.-Z. Li, "Strain replacement in an epidemic model with super-infection and perfect vaccination," Mathematical Biosciences, vol. 195, no. 1, pp. 23-46, 2005.

[19] X.-Z. Li, J. Wang, and M. Ghosh, "Stability and bifurcation of an SIVS epidemic model with treatment and age of vaccination," 
Applied Mathematical Modelling, vol. 34, no. 2, pp. 437-450, 2010.

[20] J. Wang, X. Dong, and H. Sun, "Analysis of an SVEIR model with age-dependence vaccination, latency and relapse," Journal of Nonlinear Sciences and Applications. JNSA, vol. 10, no. 7, pp. 3755-3776, 2017.

[21] M. Iannelli, Mathematical Theory of Age-Structured Population dynamics, Applied Mathematics Monographs, vol. 7, Consiglio Nazionale delle Ricerche, Giardini, Pisa, Italy, 1995.

[22] G. F. Webb, Theory of Nonlinear Age-Dependent Population Dynamics, vol. 89, Marcel Dekker, New York, NY, USA, 1985.

[23] H. L. Smith and H. R. Thieme, Dynamical systems and population persistence, vol. 118 of Graduate Studies in Mathematics, American Mathematical Society, Providence, RI, USA, 2011.

[24] J. Hale, Asymptotic Behavior of Dissipative System, vol. 25 of Mathematical Surveys and Monographs, American Mathematical Society, Providence, RI, USA, 1988.

[25] H. R. Thieme, "Uniform persistence and permanence for nonautonomous semiflows in population biology," Mathematical Biosciences, vol. 166, no. 2, pp. 173-201, 2000.

[26] P. Auger, R. Bravo de la Parra, J.-C. Poggiale, E. Sánchez, and T. Nguyen-Huu, "Aggregation of variables and applications to population dynamics," in Structured Population Models in Biology and Epidemiology, P. Magal and S. Ruan, Eds., vol. 1936 of Lecture Notes in Mathematics, pp. 209-263, Springer, Berlin, Germany, 2008. 


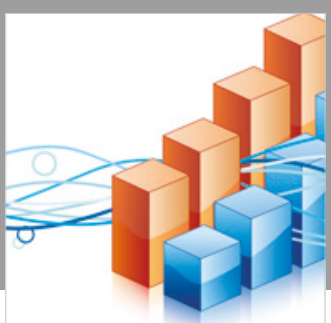

Advances in

Operations Research

\section{-n-m}
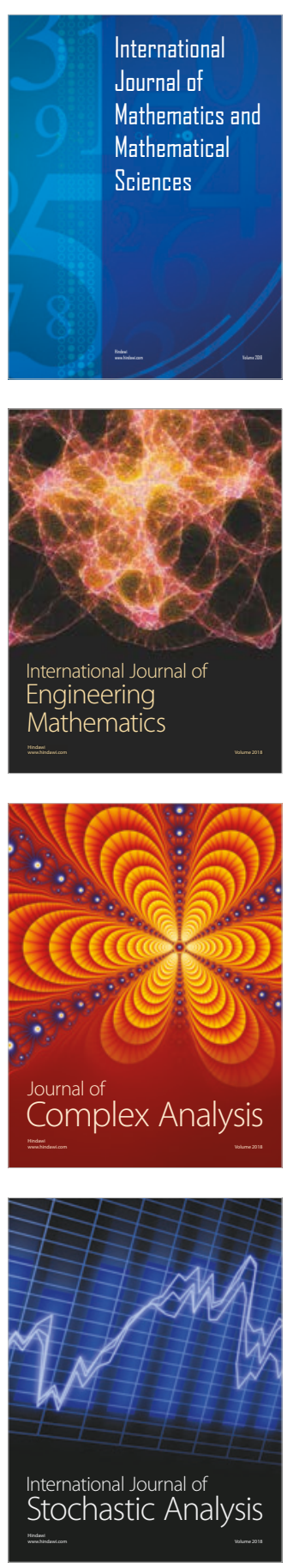
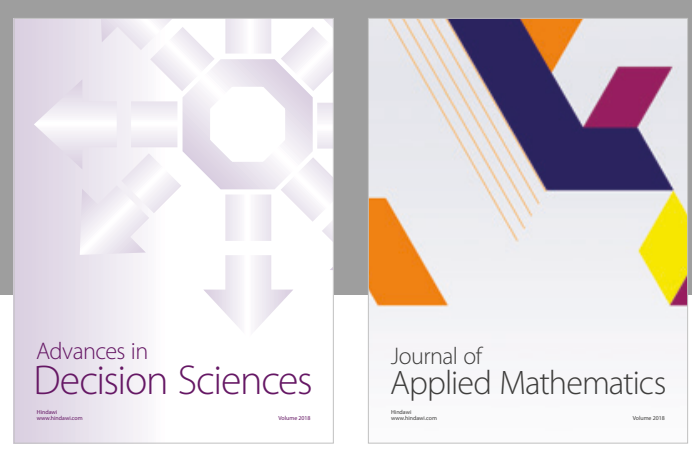

Journal of

Applied Mathematics
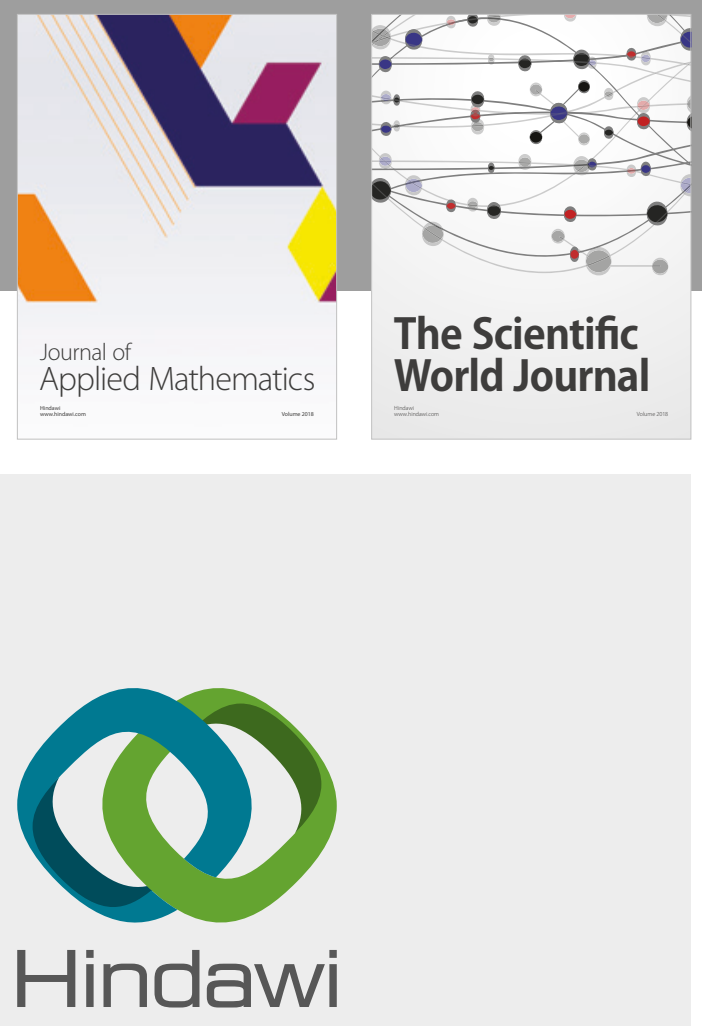

Submit your manuscripts at

www.hindawi.com

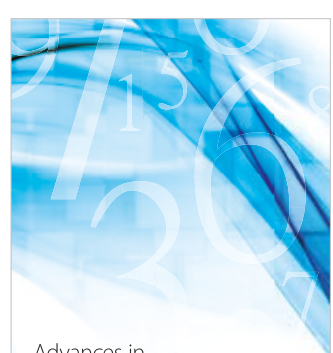

Advances in
Numerical Analysis
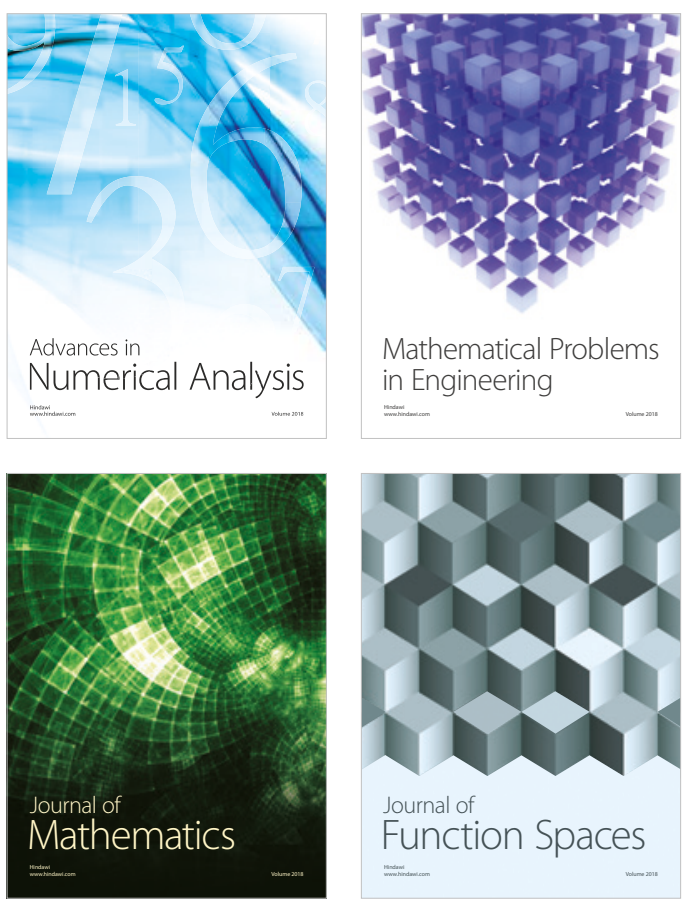

Mathematical Problems in Engineering

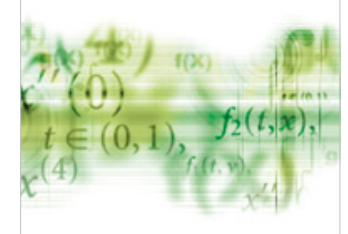

International Journal of

Differential Equations

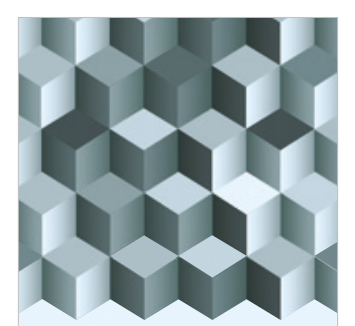

Journal of

Function Spaces

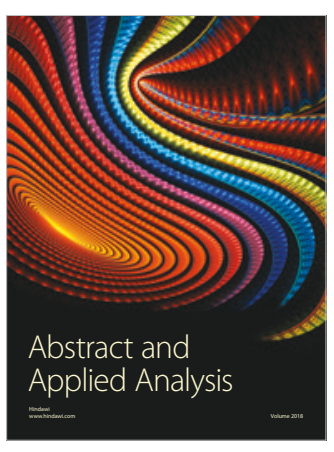

The Scientific

World Journal

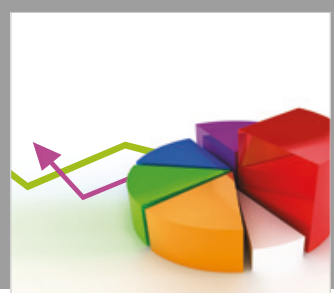

Journal of

Probability and Statistics
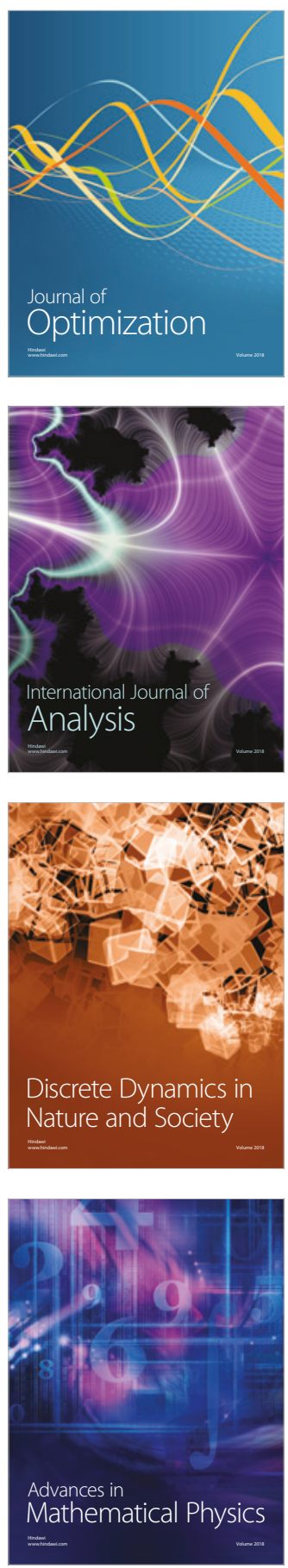